\title{
Static Highly Elliptical Orbits using Hybrid Low-Thrust Propulsion
}

\author{
Pamela Anderson ${ }^{1}$ and Malcolm Macdonald ${ }^{2}$ \\ University of Strathclyde, Glasgow, G1 1XJ, Scotland, E.U.
}

Static highly-elliptical orbits enabled using hybrid solar-sail/solar-electric propulsion are investigated. These newly proposed orbits, termed Taranis orbits, have free selection of 'critical inclination' and use low-thrust propulsion to compensate for the drift in argument of perigee caused by Earth's gravitational field. In this paper, a 12-hr Taranis orbit with an inclination of $90 \mathrm{deg}$ is developed to illustrate the principle. The acceleration required to enable this novel orbit is made up partly by the acceleration produced by solar-sails of various characteristic accelerations, and the remainder supplied by the electric thruster. Order of magnitude mission lifetimes are determined, a strawman mass budget is also developed for two system constraints, firstly spacecraft launch-mass is fixed, and secondly the maximum thrust of the thruster is constrained. Fixing maximum thrust increases mission lifetimes, and solar-sails are considered near to mid-term technologies. However, fixing mass results in negligible increases in mission lifetimes for all hybrid cases considered, solar sails also require significant development. This distinction highlights an important contribution to the field, illustrating that addition of a solar-sail to an electric propulsion craft can have negligible benefit when mass is the primary system constraint. Technology requirements are also outlined, including sizing of solar-arrays, propellant tanks and solarsails.

\footnotetext{
${ }^{1}$ PhD Candidate, Advanced Space Concepts Laboratory, pamela.c.anderson@strath.ac.uk

2 Associate Director, Advanced Space Concepts Laboratory, malcolm.macdonald.102@strath.ac.uk, Associate Fellow of the AIAA.
} 


\section{Nomenclature}

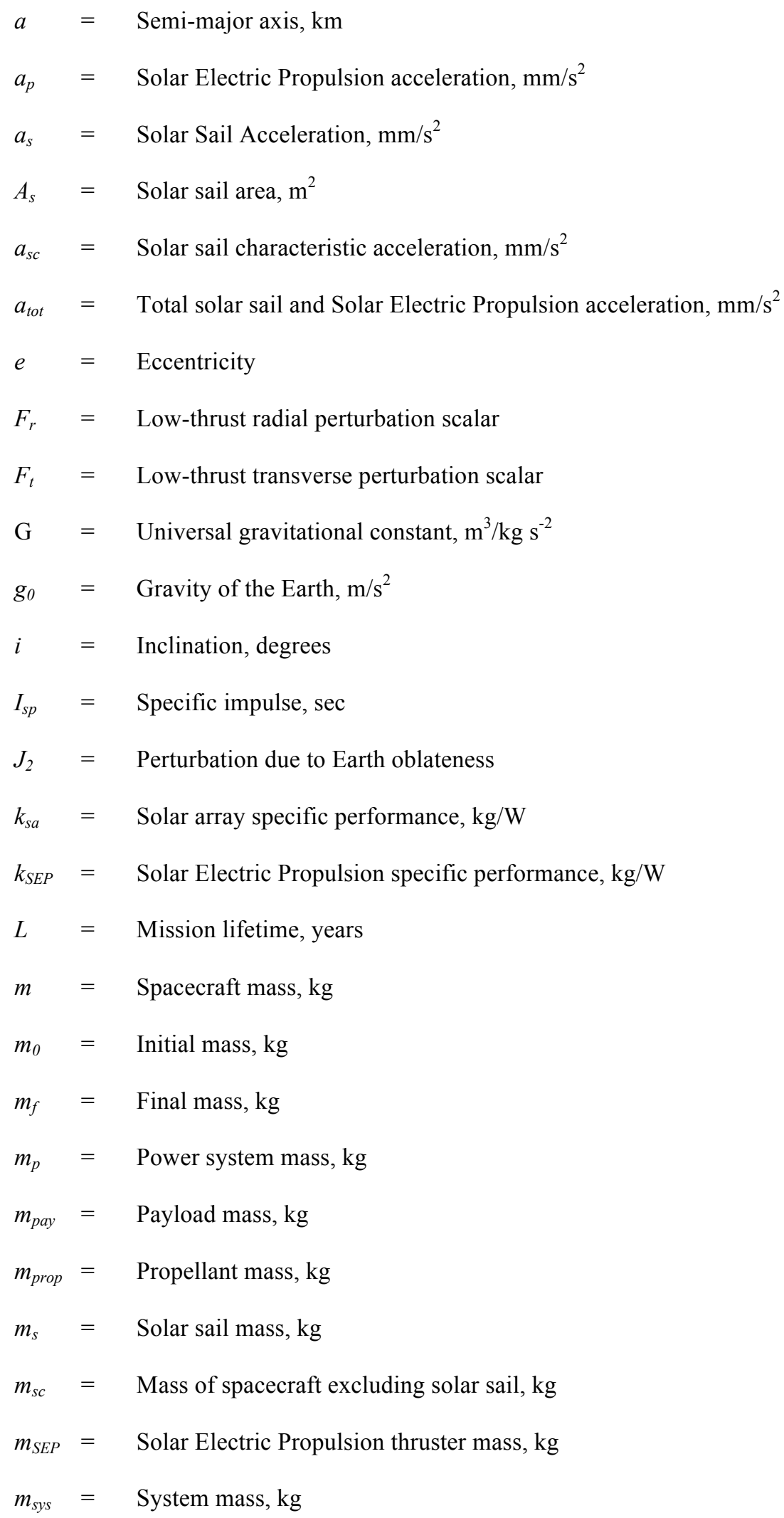




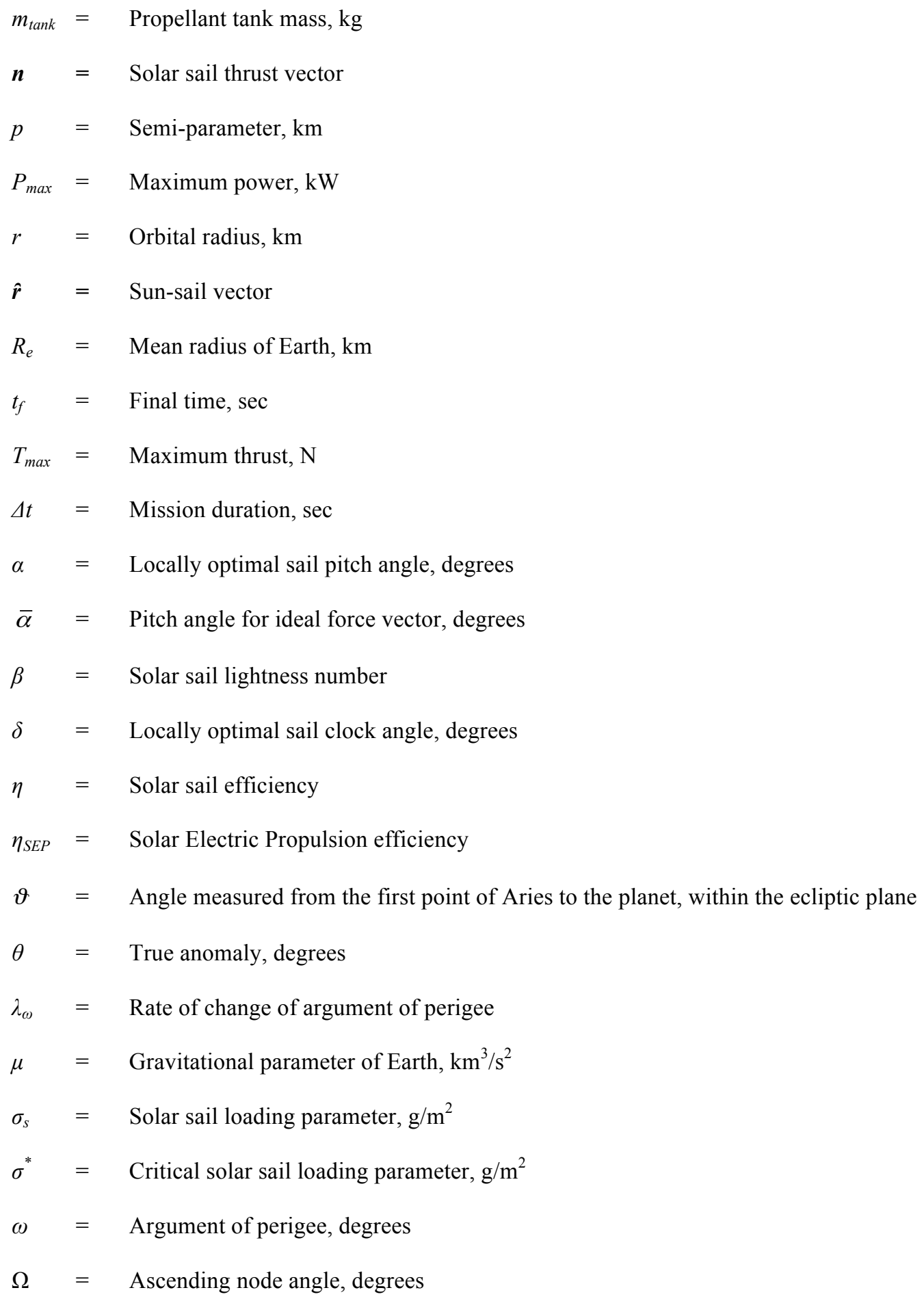

\section{Introduction}

THE extension of highly elliptical orbits (HEOs) using low-thrust propulsion to counteract the perturbations due to the Earth's gravity have previously been considered, to enable HEOs with free selection of the 'critical 
inclination' [1], with no rotation of the apsidal line [2-4]. Such orbit extensions are termed Taranis orbits [4]. One particular example is the 12-hr Taranis orbit inclined at 90deg to the equator to enable continuous observation of frigid and neighboring temperate regions. Previously, Taranis orbits have been considered using Solar Electric Propulsion (SEP), a mature technology with a high Technology Readiness Level (TRL) $[5,6]$ and low Advanced Degree of Difficulty $\left(\mathrm{AD}^{2}\right)[5,6]$. However, mission lifetimes are limited by the amount of propellant that can be carried. Consequently, consideration is given to the addition of a solar sail to the system. Solar sails exploit solar radiation pressure generated by photons reflecting off a large, highly reflective sail to produce a continuous, propellant-less thrust. Hybrid SEP / solar sail systems reduce the propellant requirements of the SEP thruster, while the SEP system compensates for the inability of the solar sail to thrust in the direction of the Sun. The use of a small solar sail on the spacecraft also contributes towards lowering the $\mathrm{AD}^{2}$ of solar sailing [5]. It is also noted that the addition of a solar sail to the system will create system level design complexities, however these effects are considered out with the scope of the paper, and are consequently neglected to allow a simple comparison to be made.

The complementary nature of the two propulsion systems enables a new range of missions in which continuous low-thrust is required. These hybrid solar sail / SEP systems have been proposed for various applications including geostationary orbits (GEO) displaced above or below the equatorial plane to create new geostationary slots [7]. Moreover, the hybrid propulsion concept has recently been proposed to modify the well-known Pole Sitter mission concept [8], enabling a spacecraft to be statically stationed high above one of the Earth's poles at over two-million kilometers range, giving continuous observation of the Earth's poles using a single spacecraft [9-11]. In each of these studies considering hybrid solar sail / SEP propulsion, either the launch mass of the spacecraft has been fixed or the maximum thrust from the SEP system has constrained the analysis. However, in this paper the comparison is made between the two constraining parameters to determine the most beneficial scenario and the development in solar sailing technology required.

This paper significantly extends the work introduced in [2-4] by considering the addition of a solar sail to the SEP system, to enable the Taranis orbits using a hybrid SEP / solar sail low-thrust propulsion system. Two cases are considered assuming different constraining parameters. Firstly, it is assumed that the launch mass of the spacecraft is fixed. Thus the mass of the solar sail added to the system is limited to be no more mass than the amount of propellant saved by the addition of the sail. Secondly, consideration is given to a system limited by the maximum 
thrust of the SEP system, thus allowing the initial mass of the spacecraft to vary. Consideration is given to allocating this additional mass in two ways, in the first instance; it is assigned solely to the solar sail. In the second instance it is used in part for the solar sail and in part to increase the useful payload capacity. The impact of the added solar sail is considered on mission lifetime along with the development of strawman mass budgets to quantify the benefit of a hybrid SEP / solar sail system over a pure SEP system.

\section{Taranis Orbits}

Taranis orbits are HEOs unconstrained by orbit period, perigee and apogee altitude or inclination. They are characterized by the application of a small continuous acceleration to ensure no rotation of the apsidal line due to the oblateness of the Earth. The total acceleration required to achieve a Taranis orbit of any inclination is obtained from the expression for the change in argument of perigee, due to $\mathrm{J}_{2}$ effects and the addition of continuous radial and transverse acceleration only [2]. It is noted that the out of plane acceleration is not included in this term, as it has been shown previously in [4] that the normal acceleration component offers limited value and significantly complicates both the analytical expressions and the spacecraft control profile.

$$
(\Delta \omega)_{0}^{2 \pi}=(\Delta \omega)_{J_{2}}+(\Delta \omega)_{F_{r}}+(\Delta \omega)_{F_{t}}
$$

where each acceleration component is given by

$$
\begin{aligned}
(\Delta \omega)_{J_{2}}= & \frac{3 J_{2} \pi R_{e}^{2}(3+5 \cos 2(i))}{4 a^{2}\left(-1+e^{2}\right)} \\
(\Delta \omega)_{F_{r}} & =\frac{1}{e \mu} 2 a^{2} F_{r}\left(-2+2 e^{2}-4 e \sqrt{-1+e^{2}} \operatorname{Arctanh}\left[\frac{-1+e}{\sqrt{-1+e^{2}}}\right]\right. \\
& \left.-e \sqrt{-1+e^{2}} \ln \left[\frac{1-e}{\sqrt{-1+e^{2}}}\right]+e \sqrt{-1+e^{2}} \ln \left[\frac{-1+e}{\sqrt{-1+e^{2}}}\right]\right) \\
(\Delta \omega)_{F_{t}} & -\frac{4 a^{2}\left(-2+e^{2}\right) F_{t}}{e \mu}
\end{aligned}
$$


The orbital elements from Table 1 are substituted into Eq. (1), for an inclination of 90deg, and the change in argument of perigee is set equal to zero results in the expression for the radial acceleration as a function of transverse acceleration. It is noted that for the orbits considered in the paper i.e. e $<1$, Eq. (3) contains complex numbers in the arguments, however when the orbital parameters from Table 1 are substituted into the equation, the acceleration required to change the argument of perigee is real.

$$
F_{r}=-1.579 e^{-4}+1.676 F_{t}
$$

The transverse acceleration is assigned a range of values between $0 \mathrm{~mm} / \mathrm{s}^{2}$ and $0.1 \mathrm{~mm} / \mathrm{s}^{2}$ and the corresponding radial acceleration is found using Eq.(5). From this, it is found that the minimum acceleration magnitude occurs when the transverse acceleration is equal to $0.07 \mathrm{~mm} / \mathrm{s}^{2}$ and the corresponding radial acceleration is $-0.0406 \mathrm{~mm} / \mathrm{s}^{2}$, this results in a total required acceleration magnitude of $0.0809 \mathrm{~mm} / \mathrm{s}^{2}$.

Table 1 Orbital parameters.

\begin{tabular}{lc}
\hline \hline & \\
Orbital Element & Value \\
\hline Perigee Altitude & $813(\mathrm{~km})$ \\
Apogee Altitude & $39540(\mathrm{~km})$ \\
Ascending Node & $330(\mathrm{deg})$ \\
Argument of perigee & $270(\mathrm{deg})$ unless otherwise stated \\
\hline \hline
\end{tabular}

\section{Hybrid SEP / Solar Sail Propulsion}

To lower the demands placed upon the SEP system, by lowering its required thrust, the use of hybrid SEP / solar sail propulsion to maintain the Taranis orbit is considered. The total acceleration needed to maintain the Taranis orbit has been shown as $0.0809 \mathrm{~mm} / \mathrm{s}^{2}$; using hybrid propulsion a fraction of this acceleration is now generated by the solar sail with the SEP system supplying the remainder of the acceleration. The total required acceleration is given by

$$
a_{t o t}=a_{s}+a_{p}
$$

where $a_{s}$ is the acceleration generated by a perfectly reflecting solar sail [12]

$$
\mathbf{a}_{\mathbf{s}}=\beta \frac{G m_{s}}{r^{2}}(\hat{\mathbf{r}} \operatorname{dot} \mathbf{n})^{2} \mathbf{n}
$$


and where $\beta$ is the dimensionless sail lightness number given as

$$
\beta=\frac{\sigma^{*}}{\sigma_{s}}
$$

The critical sail loading, $\sigma^{*}$, in Eq. (8), is equal to $1.53 \mathrm{~g} / \mathrm{m}^{2}$ [12], and the sail loading, $\sigma_{\mathrm{s}}$, is the ratio of the spacecraft mass to solar sail area

$$
\sigma_{s}=\frac{m_{s}}{A_{s}}
$$

A parallel design parameter to the sail loading is the solar sail characteristic acceleration, and is defined as the actual acceleration experienced by the sail at a solar distance of $1 \mathrm{AU}$ with the sail surface normal to the Sun, and may be written as [12]

$$
a_{s c}=\frac{2 \eta P}{\sigma_{s}}
$$

At a distance of $1 \mathrm{AU}$ from the Sun the magnitude of the solar radiation pressure, $\mathrm{P}$, exerted on a perfectly absorbing surface is $4.56 \times 10^{-6} \mathrm{~N} / \mathrm{m}^{2}$ [12]. The solar sail efficiency, $\eta$, is a function of the optical properties of the sail film and the sail shape, and accounts for the finite reflectivity of the film; typically this taken to be of the order of $0.85-0.9$ [12].

$$
a_{s c}=\frac{2 \eta P}{\sigma_{s}+\left(m_{s c} / A_{s}\right)}
$$

Equation (11) shows that the sail characteristic acceleration is a function of the spacecraft mass, $\mathrm{m}_{\mathrm{sc}}$, excluding the solar sail mass The mass of the spacecraft will decrease as the SEP thruster consumes propellant. Thus, the sail characteristic acceleration increases over time.

\section{Locally Optimal Control Laws}

Locally optimal control laws are used to maximize the instantaneous rate of change of a given orbital element and provide the required thrust orientation in analytical form [13]. Where, locally optimal refers to optimal at a given time. These locally optimal control laws are used only by the solar sail component of the force, whereas the 
SEP system uses the simple switching law previously derived [4]. In the absence of any low-thrust propulsion to maintain the 12-hr Taranis orbit, the natural drift in argument of perigee due to the gravitational perturbation is $0.15 \mathrm{deg}$ per day (towards the ascending node). Thus, it is required that the sail attempt to impart positive rate of change of argument of perigee to compensate for this drift. The argument of perigee locally optimal control law is derived from the argument of perigee variational equation, given in terms of classical orbital elements

$$
\frac{d \omega}{d \theta}=\left[\begin{array}{lll}
R & T & N
\end{array}\right] \lambda_{\omega}
$$

where

$$
\lambda_{\omega}=\left[\begin{array}{c}
-\frac{\sqrt{p}}{e} \cos \theta \\
\left(1+\frac{r}{p}\right) \frac{\sqrt{p}}{e} \sin \theta \\
-\frac{r}{\sqrt{p}} \cot i \sin (\theta+\omega)
\end{array}\right]
$$

The locally optimal control law thus requires the solar sail thrust to be maximized along $\lambda_{\omega}$, [13]. The solar sail trajectory is planet-centered; therefore $\lambda_{\omega}$ must be transposed into the Sun-sail line reference frame, shown in Fig. 1, using standard transformation matrices. The transformation is performed in two stages; first, transformation from planet-centered RTN to Earth-centered inertial using the inverse of the transformation matrix [14]

$$
\begin{aligned}
& O_{R T N}=[T] O_{E C I} \\
& =\left[\begin{array}{ccc}
\cos (\omega+\theta) \cos \Omega-\sin (\omega+\theta) \cos i \sin \Omega & \cos (\omega+\theta) \sin \Omega+\sin (\omega+\theta) \cos i \cos \Omega & \sin (\omega+\theta) \sin i \\
-\sin (\omega+\theta) \cos \Omega-\cos (\omega+\theta) \cos i \sin \Omega & -\sin (\omega+\theta) \sin \Omega+\cos (\omega+\theta) \cos i \cos \Omega & \cos (\omega+\theta) \sin i \\
\sin i \sin \Omega & -\sin i \cos \Omega & \cos i
\end{array}\right] O_{E C I}
\end{aligned}
$$




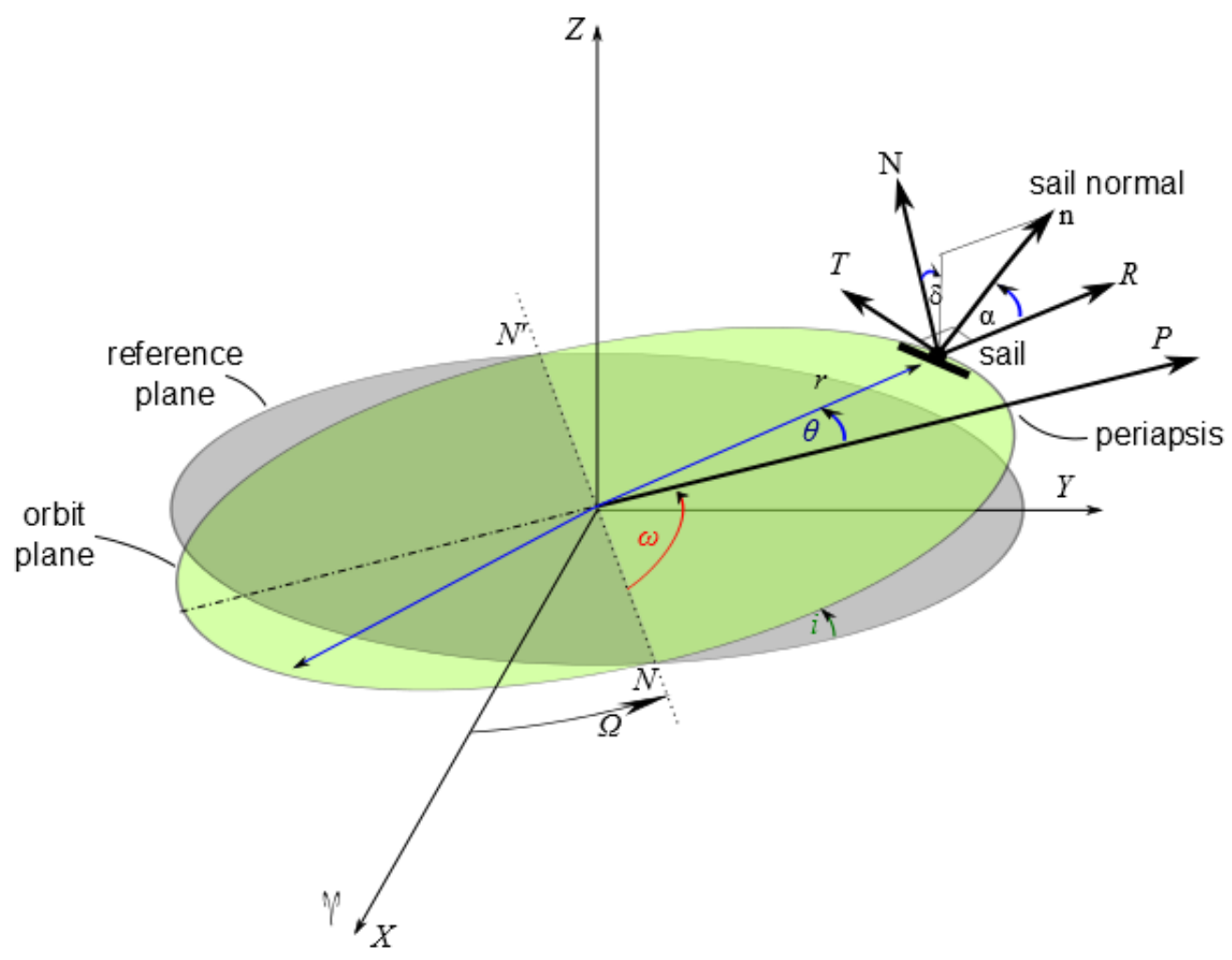

Fig. 1 Orientation of solar sail pitch and clock angles.

The second stage is to transform from Earth-centered inertial to Sun-line coordinates, using the transformation matrix given in Eq.(15) [14]. It is noted that orthogonal coordinate systems are employed and that parallax effects are negligible.

$$
O_{S U N}=[T] O_{E C I}=\left[\begin{array}{ccc}
\cos \vartheta & \sin \vartheta \cos \varepsilon & \sin \vartheta \sin \varepsilon \\
-\sin \vartheta & \cos \vartheta \cos \varepsilon & \cos \vartheta \sin \varepsilon \\
0 & -\sin \varepsilon & \cos \varepsilon
\end{array}\right] O_{E C I}
$$

With conversion of $\lambda_{\omega}$ into the Sun-sail line coordinate system, the pitch angle of the ideal force vector is found using Fig. 1, and is given as

$$
\tilde{\alpha}=\arccos \left(\lambda_{\hat{x}}^{s s}\right)
$$

where, $\hat{\lambda}_{\omega}=\left[\begin{array}{lll}\lambda_{\omega x}^{s s} /\left|\lambda_{\omega}\right| & \lambda_{\omega y}^{s s} /\left|\lambda_{\omega}\right| & \lambda_{\omega z}^{s s} /\left|\lambda_{\omega}\right|\end{array}\right]=\left[\begin{array}{lll}\hat{\lambda}_{\omega x}^{s s} & \hat{\lambda}_{\omega y}^{s s} & \hat{\lambda}_{\omega z}^{s s}\end{array}\right]$, the unit argument of perigee vector taken from the RTN co-ordinate system to the Sun-sail line coordinate system. Hence superscripts ss refer to the Sun-sail co- 
ordinate system and subscripts $\mathrm{x}, \mathrm{y}$, and $\mathrm{z}$ refer to the $\mathrm{x}, \mathrm{y}$, and $\mathrm{z}$ components of the argument of perigee vector. The sail orientation to maximize the sail thrust vector is found, the locally optimal sail pitch angle is given from [15]

$$
\tan \alpha=\frac{-3 \cos \tilde{\alpha}+\sqrt{9 \cos ^{2}(\tilde{\alpha})+8 \sin ^{2}(\tilde{\alpha})}}{4 \sin \tilde{\alpha}}
$$

The locally optimal sail clock angle is also found using Fig. 1 and is given as

$$
\delta=\arccos \left(\frac{\lambda_{\bar{z}}}{\sqrt{\lambda_{\hat{y}}^{2}+\lambda_{z}^{2}}}\right)
$$

The solar sail thrust vector in Eq. (7) is found using [12]

$$
\mathbf{n}=\left[\begin{array}{l}
\cos \alpha \\
\sin \alpha \sin \delta \\
\sin \alpha \cos \delta
\end{array}\right]
$$

Where $\alpha$ is the sail pitch angle, or the angle between the sail normal and incident radiation, and $\delta$ is the sail clock angle, the angle measured within a plane normal to the Sun line from the projection of the orbit normal vector in that plane. As the sail is in an Earth-centered trajectory the normal vector orientation given in Eq. (19) must be transformed into planet-centered RTN coordinates. This is again performed in two stages transforming from Sunline coordinates to Earth-centered inertial, using the inverse of the transformation matrix given in Eq. (15), and from Earth-centered inertial to Earth-centered RTN, using the matrix in Eq. (14).

General perturbations methods are used to determine the total acceleration required to enable the Taranis orbits. Special perturbations methods use these acceleration values and numerically integrate the Lagrange planetary equations including the motion of the solar sail. Using the defined solar sail equations, various solar sail characteristic accelerations are considered to determine the sail characteristic acceleration that offers the most benefit in terms of the lowest acceleration demand on the SEP system. As the characteristic acceleration of the sail is increased, at some point the acceleration provided by the solar sail will be so high that the SEP system will be required to counteract the sail acceleration, rather than supplement it. Using a spacecraft with an initial mass of $1000 \mathrm{~kg}$ and considering four values of solar sail characteristic accelerations of $0.03,0.05,0.07$, and $0.1 \mathrm{~mm} / \mathrm{s}^{2}$, the 
mass of the spacecraft is shown in Fig. 2 over the first year of operation, the remaining mass is also shown in Fig. 3 as a function of the solar sail characteristic acceleration over various time intervals.

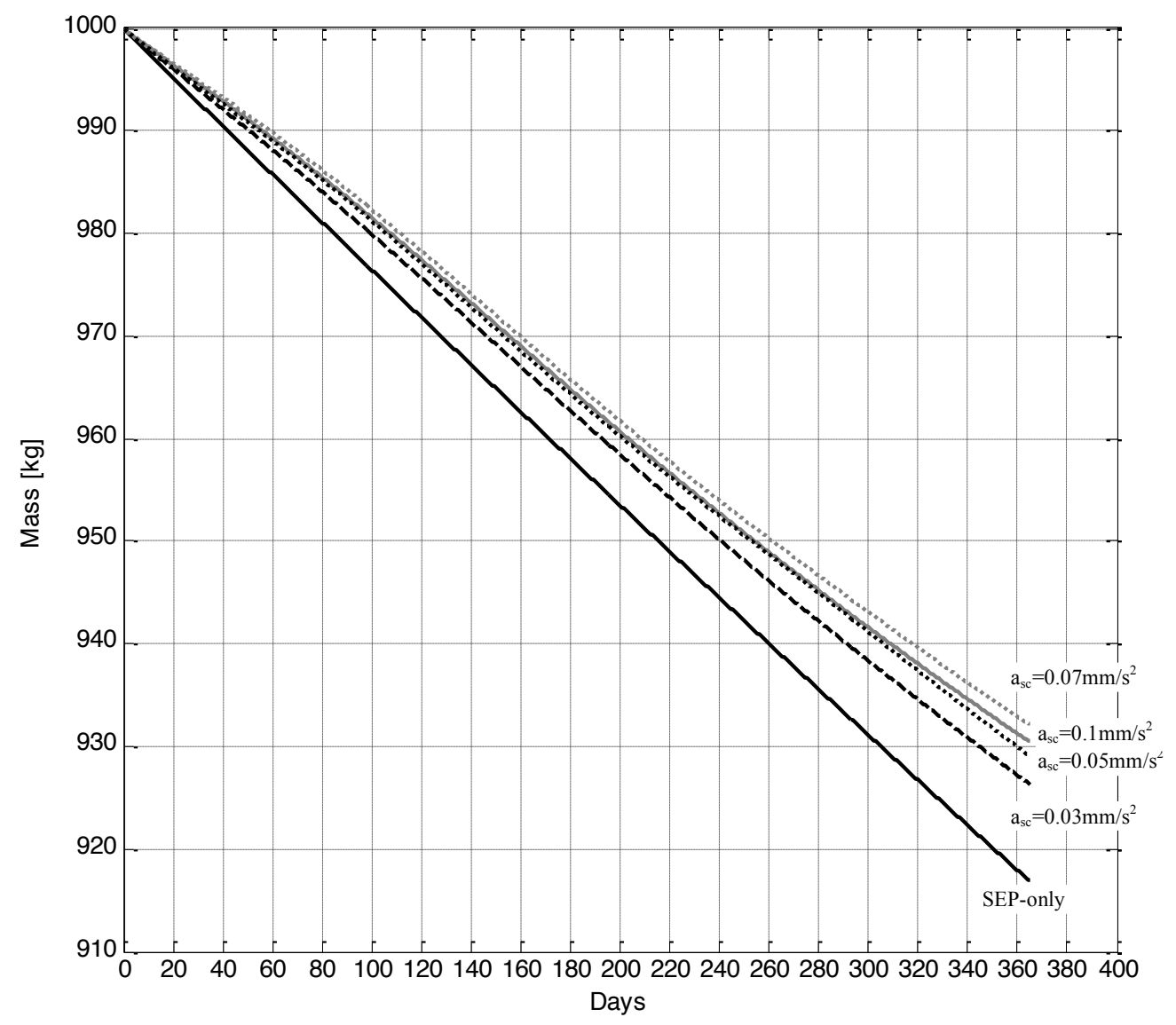

Fig. 2 Fuel consumption of spacecraft over one year using various solar sail characteristic accelerations. 


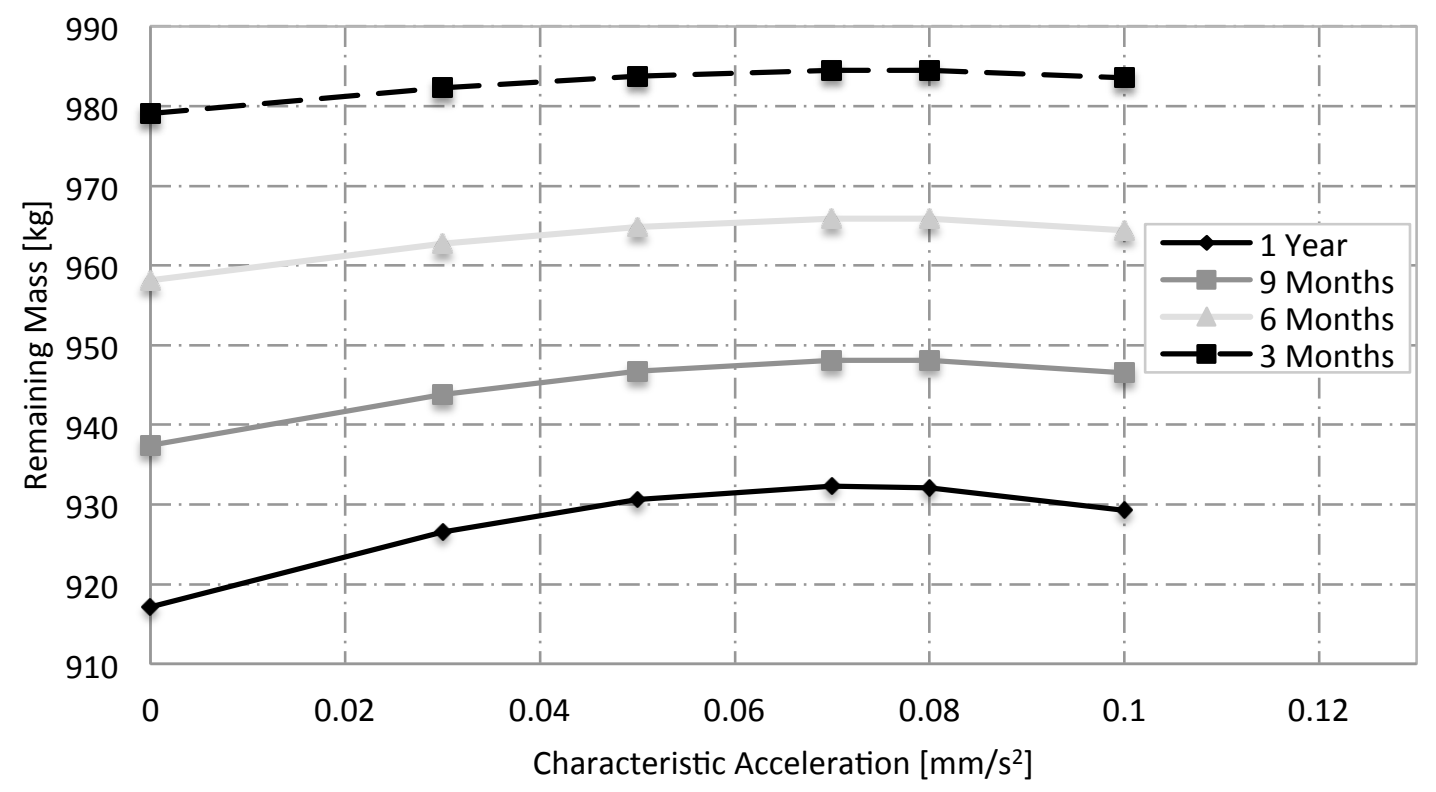

Fig. 3 Remaining mass as a function of characteristic acceleration.

It is seen from Fig. 2 that, as expected, each solar sail considered offers some benefit over the SEP-only case, by decreasing the amount of propellant consumed, thus increasing the final spacecraft mass. The solar sail with a characteristic acceleration of $0.07 \mathrm{~mm} / \mathrm{s}^{2}$ offers the highest reduction in fuel consumption. This turning point was determined by a process of trial and error, increasing the characteristic acceleration in increments of $0.001 \mathrm{~mm} / \mathrm{s}^{2}$. Increasing the characteristic acceleration above this value causes the SEP system to counteract the acceleration generated by the solar sail, thus increasing the fuel consumption of the SEP system. This turning point will, however, move as the SEP system uses up propellant and the solar sail acceleration increases, as in Eq (11). The maximum propellant mass saving over the first year, given by a sail characteristic acceleration of $0.07 \mathrm{~mm} / \mathrm{s}^{2}$, is just over $15 \mathrm{~kg}$. With characteristic accelerations of $0.03 \mathrm{~mm} / \mathrm{s}^{2}$ and $0.05 \mathrm{~mm} / \mathrm{s}^{2}$ offering reductions in propellant consumption of around $9 \mathrm{~kg}$ and $13.5 \mathrm{~kg}$ respectively.

The acceleration provided by the solar sail varies throughout the year due to the tilt of the Earth's rotational axis with respect to the orbit plane, thus the acceleration required by the SEP system varies throughout the year, the necessary SEP acceleration is shown in Fig. 4. 


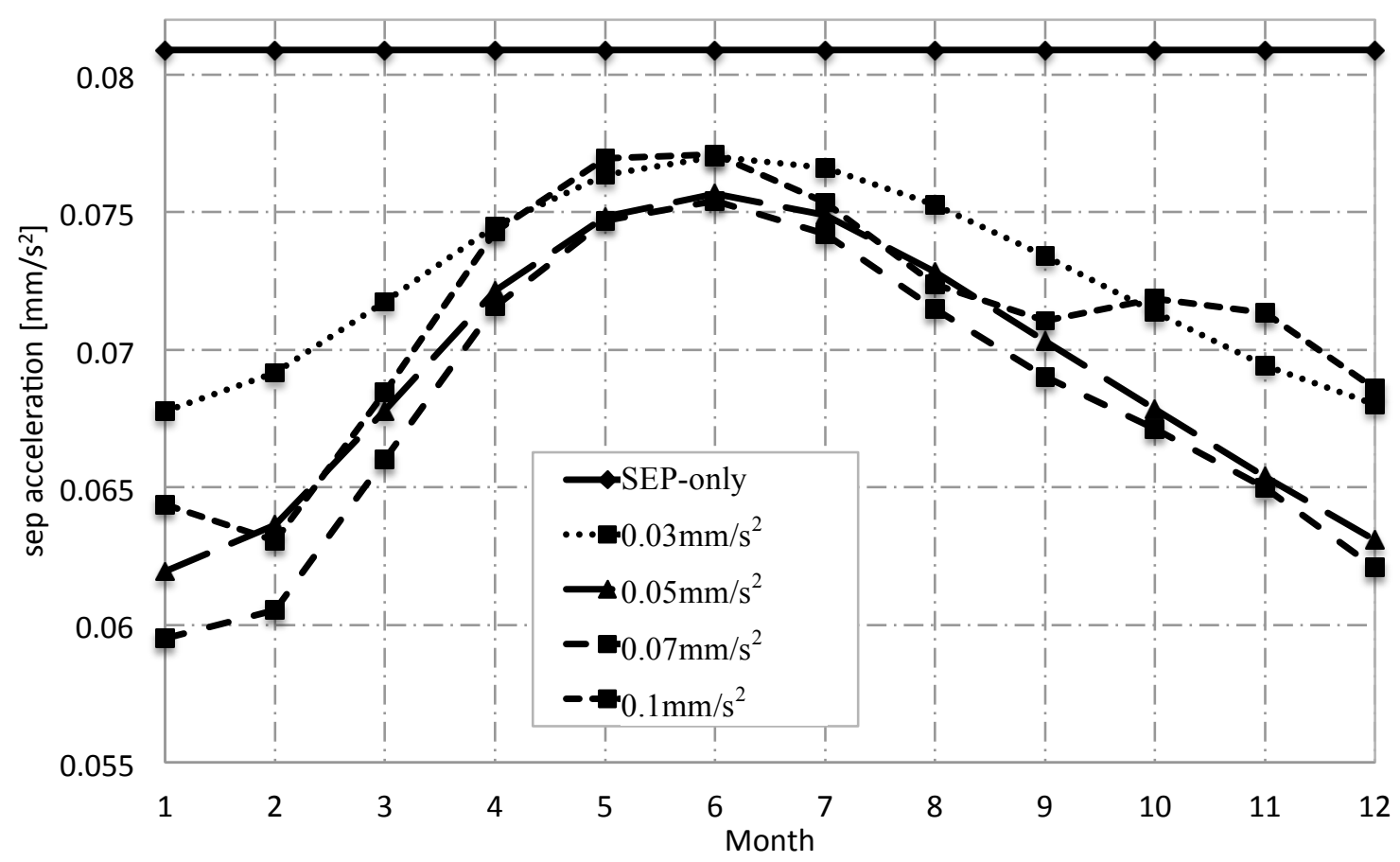

Fig. 4 SEP acceleration required over one year.

Fig. 4 shows that from April to August the acceleration from the sail decreases, thus the maximum demand on the SEP system occurs during this time. A significant decrease in the required SEP acceleration is shown throughout the Northern Hemisphere winter. The turning point described in Fig. 2 is again explained in Fig. 4, where it is shown that the acceleration generated by the solar sail with a characteristic acceleration of $0.1 \mathrm{~mm} / \mathrm{s}^{2}$ is so high that the SEP thruster has to counteract this.

The solar sail control angles required to achieve the 90deg inclination Taranis orbits and accomplish the stated reduction in total SEP accelerations are shown in Fig. 5 and Fig. 6. 


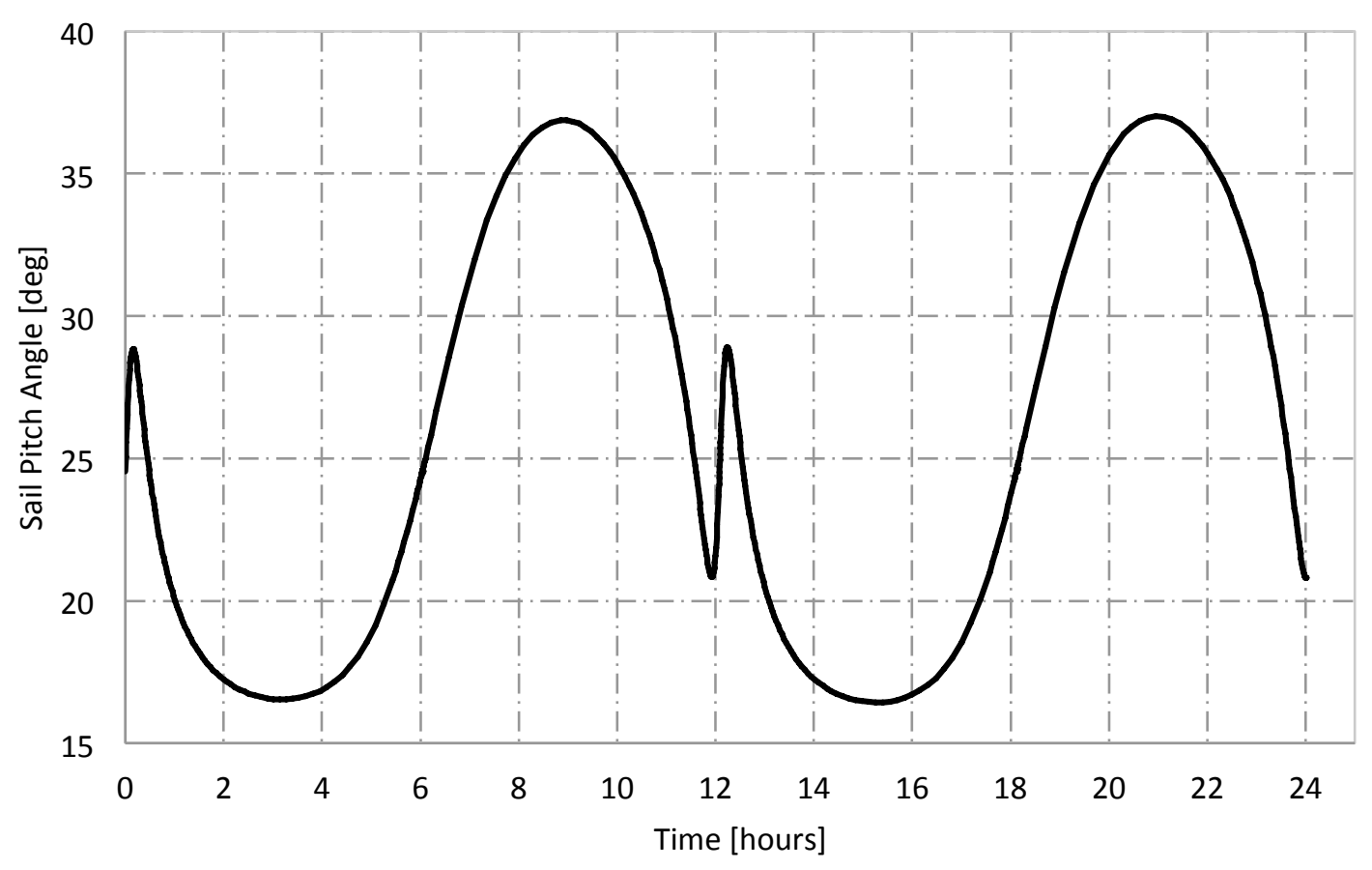

Fig. 5 Solar Sail Pitch Angle.

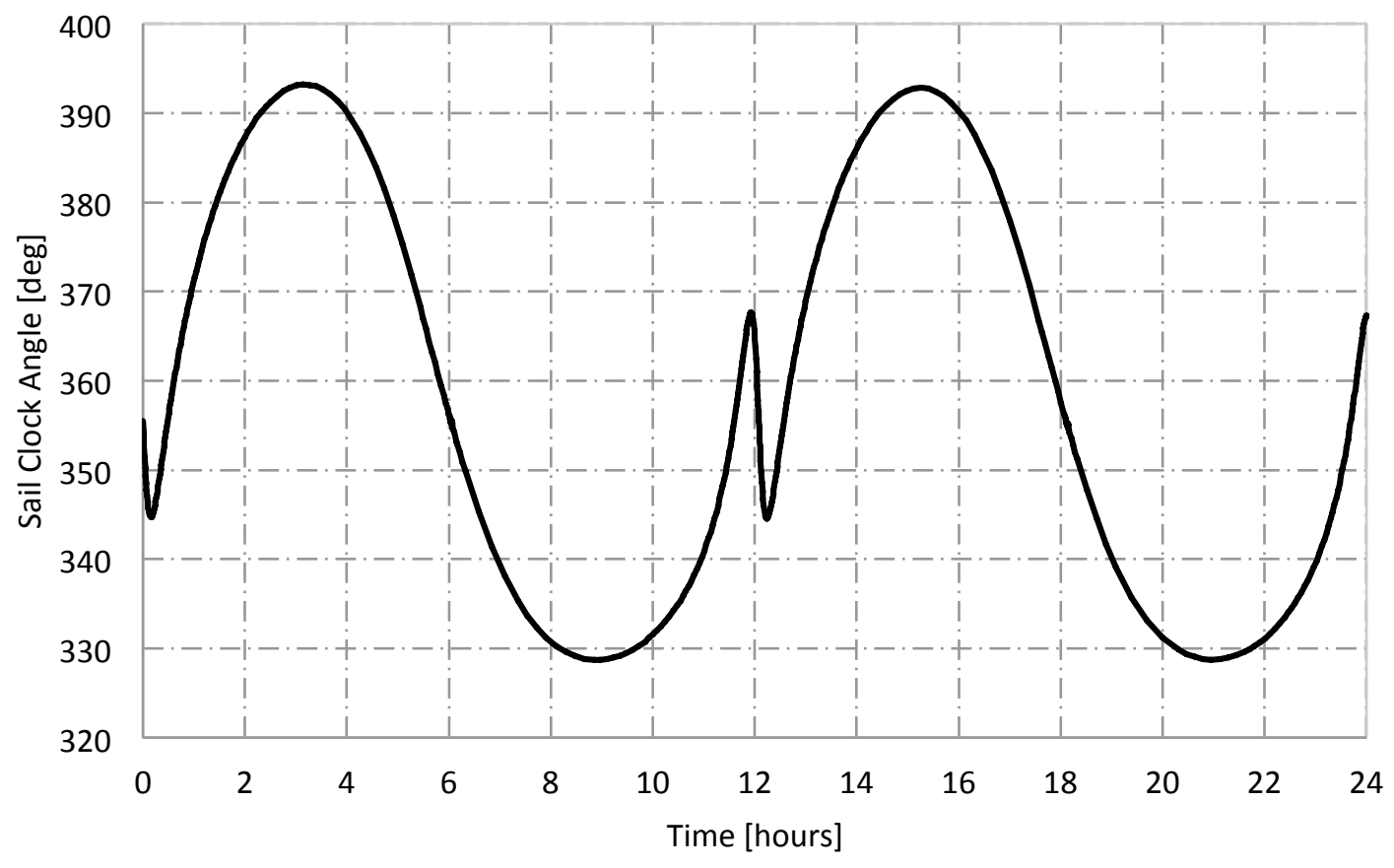

Fig. 6 Solar Sail Clock Angle.

From Fig. 5 it is shown that the sail pitch angle must rotate by around 20deg per orbit, while from Fig. 6 the sail clock angle has been plotted from -180deg to $180 \mathrm{deg}$ to show the motion is smooth, the sail clock angle therefore 
completes three $360 \mathrm{deg}$ revolutions within 12-hrs. The relatively short orbit period of the Taranis orbits, means that fast slew maneuvers are required by the solar sail, which are currently beyond the current capabilities of solar sail technology.

\section{Mission Analysis}

\section{Mission Lifetime}

By evaluating the performance of the 12-hr Taranis orbit in terms of propellant consumption, possible mission lifetimes of the orbit facilitated by means of SEP-only and hybrid SEP/solar sail systems are determined. In the hybrid SEP/solar sail systems the constant acceleration is given by taking the average acceleration required by the SEP system over the first year, given in Table 2.

$$
L=t_{f}=-\ln \left(\frac{m_{f}}{m_{0}}\right) \frac{I_{s p} g_{0}}{a_{p}}
$$

where, the mass fraction $\mathrm{m}_{\mathrm{f}} / \mathrm{m}_{0}$ is defined as

$$
\frac{m_{f}}{m_{0}}=\frac{\left(m_{0}-m_{\text {prop }}\right)}{m_{0}}
$$

Table 2 Average SEP Acceleration over a 12 month period.

\begin{tabular}{cc}
\hline \hline $\begin{array}{c}\text { Characteristic Acceleration } \\
{\left[\mathbf{m m} / \mathbf{s}^{2}\right]}\end{array}$ & $\begin{array}{c}\text { Average SEP Acceleration } \\
{\left[\mathbf{m m} / \mathbf{s}^{2}\right]}\end{array}$ \\
\hline $\begin{array}{c}\text { SEP-only } \\
0.03\end{array}$ & 0.0809 \\
0.05 & 0.0726 \\
0.07 & 0.0692 \\
0.10 & 0.0681 \\
\hline
\end{tabular}

It is noted from Table 2 that the acceleration required from the SEP system decreases by less than the sail characteristic acceleration, this is due to the useful sail acceleration magnitude being set by the square of the cosine of the pitch angle. The lifetime of the 90deg, 12-hr Taranis orbit, detailed in Table 1, is thus determinable for a particular mass fraction and specific impulse. The resulting possible mission lifetimes are shown in Fig. 7 for the SEP-only system and for each of the hybrid propulsion systems considered. 


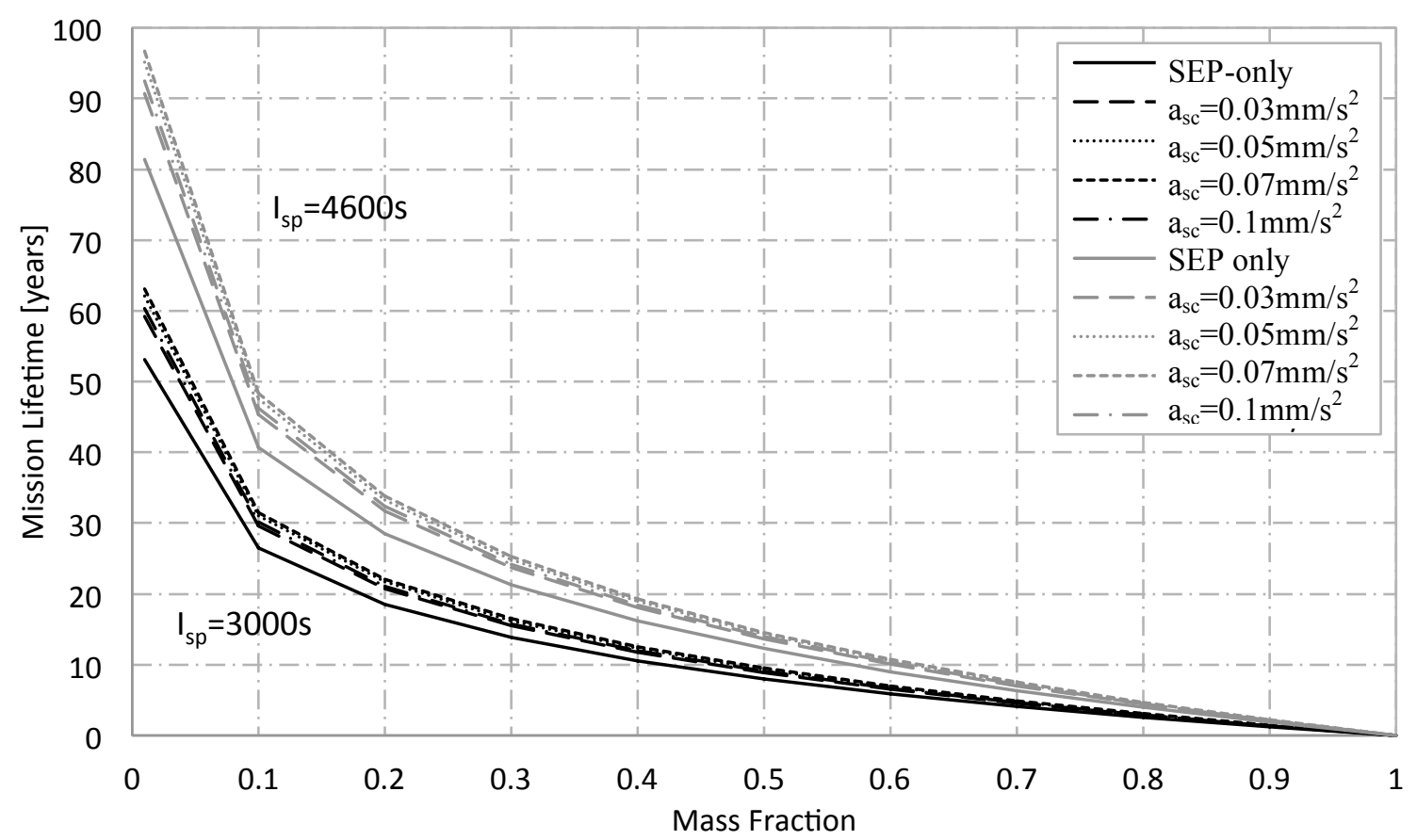

Fig. 7 Taranis mission lifetime as a function of mass fraction for SEP-only and various solar sail characteristic accelerations.

Fig. 7 shows the possible mission lifetimes for the Taranis orbit as a function of mass fraction for specific impulses of 3000s and 4600s, using SEP / sail systems with various solar sail characteristic accelerations. For example, using a mass fraction of 0.5 the resulting mission lifetime for each system and both specific impulses is given in Table 3.

Table 3 Mission lifetime.

\begin{tabular}{ccc}
\hline \hline Characteristic Acceleration $\left[\mathbf{m m} / \mathbf{s}^{2}\right.$ ] & $\begin{array}{c}\text { Isp }=3000 \mathrm{~s} \\
\text { Lifetime [years] }\end{array}$ & $\begin{array}{c}\text { Isp=4600s } \\
\text { Lifetime [years] }\end{array}$ \\
\hline SEP-Only & 8.0 & 12.3 \\
0.03 & 9.0 & 13.7 \\
0.05 & 9.3 & 14.3 \\
0.07 & 9.5 & 14.6 \\
0.10 & 9.1 & 13.9 \\
\hline \hline
\end{tabular}

Table 3 shows that employing a solar sail with a characteristic acceleration of $0.07 \mathrm{~mm} / \mathrm{s}^{2}$ can enable a mission around a year and a half longer than a Taranis orbit enabled using a pure SEP system, with a specific impulse of 3000s. It is also shown that an increase in lifetime of over two years is possible increasing the specific impulse to 4600s. This highlights the benefit of the hybrid solar sail / SEP system due to the reduced propellant consumption 
from the SEP-only system. It is noted that this is a conservative analysis, as the sail will become more effective as the SEP propellant is depleted and hence the mission lifetimes will in fact be longer than detailed here.

\section{Initial Spacecraft Mass}

The maximum allowable initial mass of the spacecraft is determined for the given level of thrust, using the constant SEP accelerations given in Table 2; the maximum allowable mass is shown in Fig. 8, determined using the following expression

$$
T_{\max }=m_{0} a_{p}
$$

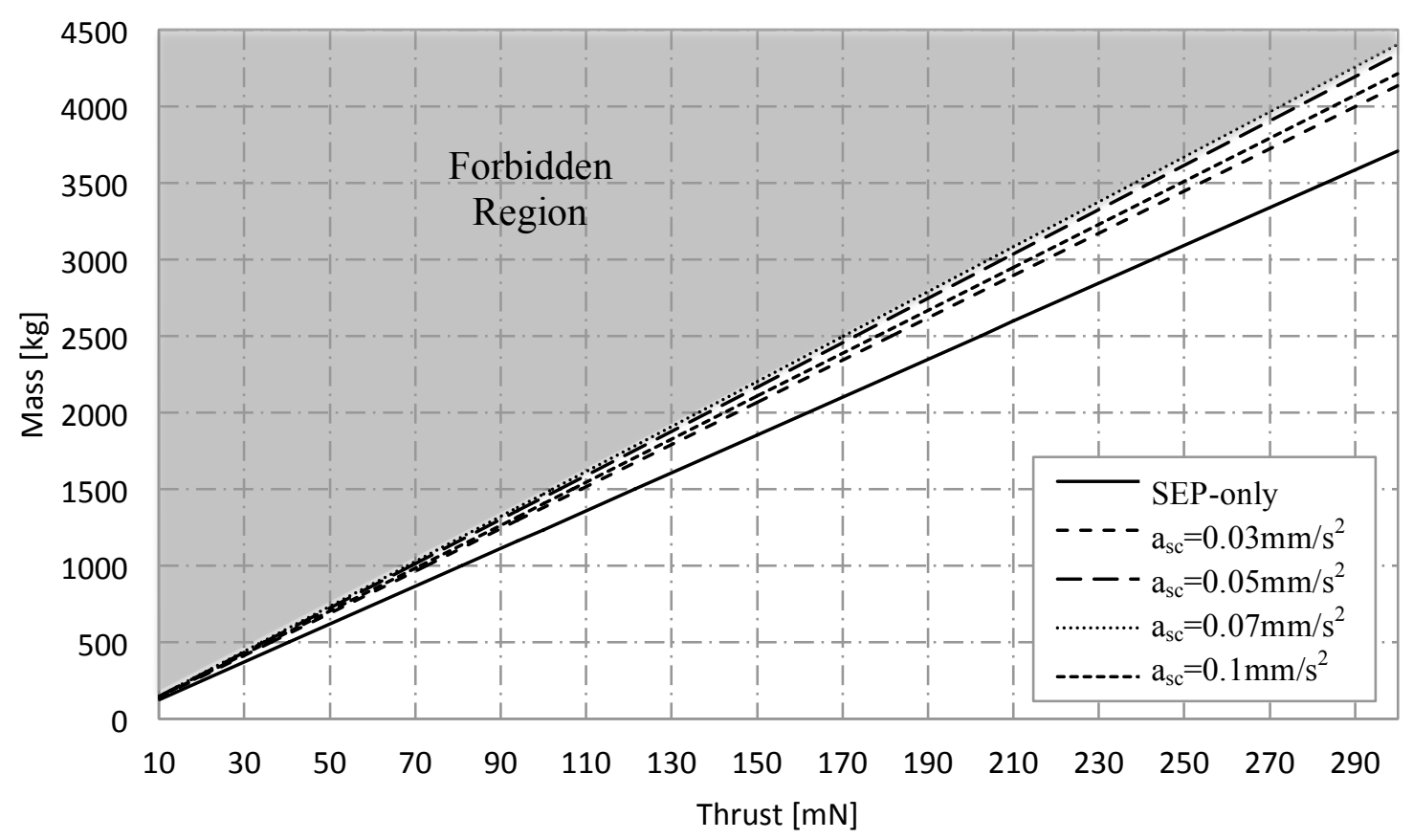

Fig. 8 Maximum allowable initial mass for the 12-hr Taranis orbit in Table 1.

Fig. 8 shows the valid region for the initial mass of the spacecraft, where the forbidden region is the area where the thrust / mass ratio is less than that required to maintain the orbit. Two separate factors can constrain the system; firstly, where the launch mass of the spacecraft is fixed and secondly where the maximum thrust of the SEP system is used to constrain the mission. This paper considers both cases to examine the possible benefits of each. 


\section{Fixed Launch Mass}

Firstly, the case where the launch mass of the spacecraft is fixed is considered. Selecting three initial masses of spacecraft of $1000 \mathrm{~kg}, 1500 \mathrm{~kg}$ and $2500 \mathrm{~kg}$ the corresponding required initial thrust values are given in Table 4.

Table 4 Maximum initial thrust values.

\begin{tabular}{cccc}
\hline \hline & $1000 \mathrm{~kg}$ & $1500 \mathrm{~kg}$ & $2500 \mathrm{~kg}$ \\
Characteristic Acceleration [mm/ $\mathbf{s}^{\mathbf{2}}$ ] & Initial Thrust [mN] & $\begin{array}{c}\text { Initial Thrust [mN] } \\
\text { Initial Thrust [mN] }\end{array}$ \\
\hline SEP-Only & 80.9 & 121.4 & 202.3 \\
0.03 & 72.6 & 108.9 & 181.5 \\
0.05 & 69.2 & 103.8 & 173.0 \\
0.07 & 68.1 & 102.2 & 170.3 \\
0.10 & 71.2 & 106.8 & 178.0 \\
\hline \hline
\end{tabular}

Table 4 shows that for a particular initial mass of spacecraft, the addition of a solar sail, reduces the thrust required by the SEP thruster. With the maximum reduction in thrust of $32 \mathrm{mN}$, occurring for the $2500 \mathrm{~kg}$ spacecraft with a solar sail with a characteristic acceleration of $0.07 \mathrm{~mm} / \mathrm{s}^{2}$ characteristic acceleration solar sail. Note that the additional mass incurred by adding a solar sail remains constrained by the fixed launch mass. As such, the solar sail mass is constrained to be no more than the saving in propellant mass for a given mission lifetime.

\section{Fixed Maximum Thrust}

The second case considers the maximum thrust of the SEP system as the constraining parameter. The maximum allowable initial mass of the spacecraft for a particular thrust value is found using Fig. 8, with the allowable mass shown to increase for each of the hybrid systems considered. For example, fixing the available SEP thrust at three particular values of $94 \mathrm{mN}, 150 \mathrm{mN}$, and $210 \mathrm{mN}$ corresponding to the thrust available from NASA's Solar Electric Propulsion Technology Application Readiness (NSTAR) thruster [16], the qualified thrust of the QinietiQ T6 thruster, and the maximum thrust of the QinetiQ T6 thruster [17] respectively, results in the maximum allowable mass values given in Table 5.

Table 5 Maximum allowable mass values.

\begin{tabular}{cccc}
\hline \hline & $94 \mathrm{mN}$ & $150 \mathrm{mN}$ & $210 \mathrm{mN}$ \\
Characteristic Acceleration $\left[\mathbf{m m} / \mathbf{s}^{\mathbf{2}}\right.$ ] & Initial Mass [kg] & $\begin{array}{c}\text { Initial Mass [kg] } \\
\text { Initial Mass [kg] }\end{array}$ \\
\hline SEP-Only & 1162 & 1854 & 2596 \\
0.03 & 1295 & 2066 & 2893 \\
0.05 & 1358 & 2167 & 3035 \\
0.07 & 1380 & 2203 & 3084 \\
0.10 & 1320 & 2107 & 2949 \\
\hline
\end{tabular}

Table 5 shows that the addition of solar sails can offer a significant increase in the allowable mass of the spacecraft. The maximum allowable mass is given using a solar sail of $0.07 \mathrm{~mm} / \mathrm{s}^{2}$ with considerable increases in 
mass of around $218 \mathrm{~kg}, 349 \mathrm{~kg}$ and $488 \mathrm{~kg}$ for each available thrust. In the first instance this additional mass is considered to be the total mass of the solar sail added to the system. It is noted that this case will produce no increase in mission lifetime over the SEP-only case, as there is no additional propellant on board. However, the relevance of including this case is that it provides the base line solar sail technology. To produce any addition in mission lifetime, solar sail performance will have to be improved beyond this. In the second instance, the additional mass is used to add an advanced light-weight solar sail to the system, providing the same characteristic acceleration and to allow additional SEP propellant useful payload to be carried.

\section{Mass Budget}

Although the mission lifetime analysis characterizes possible mission lifetimes of the Taranis mission in terms of propellant consumption, it should also be investigated whether these conditions allow a useful payload to be carried using a realistic solar sail. The initial mass of the spacecraft is composed of many elements [9]

$$
m_{0}=m_{\mathrm{sys}}+m_{\mathrm{prop}}+m_{\mathrm{tank}}+m_{\mathrm{SEP}}+m_{\mathrm{P}}+m_{\mathrm{pay}}+m_{s}
$$

From Eq.(23), the total mass of the onboard systems, $\mathrm{m}_{\mathrm{sys}}$, including data processing, telecommunications, guidance, navigation and control, structural mass, and any power system requirements beyond the SEP system requirements are assumed to total $500 \mathrm{~kg}$. The mass of the SEP thruster, $\mathrm{m}_{\mathrm{SEP}}$, is found as a function of the maximum power provided by the system, $\mathrm{P}_{\max }$, and is given by

$$
m_{\mathrm{SEP}}=k_{S E P} P_{\max }
$$

With the specific performance of the thruster given as $\mathrm{k}_{\mathrm{SEP}}=0.02 \mathrm{~kg} / \mathrm{W}[18]$, and the maximum power

$$
P_{\max }=\frac{T_{\max } I_{S P} g_{0}}{2 \eta_{S E P}}
$$

The thruster efficiency, $\eta_{\mathrm{SEP}}$, is assumed to be equal to 0.7 . In Eq.(23), $\mathrm{m}_{\mathrm{p}}$ is the mass of the spacecraft power system required to provide electrical energy to the SEP system. Thus, using a solar array the mass is given by

$$
m_{\mathrm{P}}=k_{S A} P_{\max }
$$


Using a conservative estimate of the specific performance of the solar array, from [19], of $\mathrm{k}_{\mathrm{SA}}=1 / 45 \mathrm{~kg} / \mathrm{W}$. The mass of the propellant, $\mathrm{m}_{\text {prop}}$, is found as a function of the mission duration, and is given by

$$
m_{\text {prop }}=\frac{T_{\max }}{I_{S P} g_{0}} \Delta t
$$

The mass of the propellant tanks, $\mathrm{m}_{\mathrm{tank}}$, is a function of the mass of the propellant, $\mathrm{m}_{\mathrm{tank}}=0.1 \mathrm{~m}_{\text {prop}}$. Inserting the appropriate values into Eq. (23) to find the mass of the sub-systems, the remaining mass is the useful payload mass, $\mathrm{m}_{\text {pay }}$, for a range of mission lifetimes. This process is again conducted for both the fixed launch mass and fixed maximum thrust cases.

Fixed Launch Mass

The maximum solar sail masses that can be added to the spacecraft, for the fixed launch mass case, are determined from the amount of propellant saved through the use of a hybrid low-thrust propulsion system (Fig. 2). The propellant mass saving per year in orbit for the three given solar sail characteristic accelerations are given in Table 6.

Table 6 Propellant mass saving per year.

\begin{tabular}{cc}
\hline \hline & \\
Characteristic Acceleration $\left[\mathbf{m m} / \mathbf{s}^{\mathbf{2}}\right]$ & Mass Saving [kg] \\
\hline 0.03 & 9.44 \\
0.05 & 13.56 \\
0.07 & 15.25 \\
0.10 & 12.22 \\
\hline \hline
\end{tabular}

The maximum mass of the solar sail is thus given by the propellant mass saving per year multiplied by the lifetime of the mission. In this instance, the mission lifetimes used to size the solar sails are given by the SEP-only case. Where, mission lifetimes are 4.49 years, 6.24 years, and 7.63 years for the $1000 \mathrm{~kg}, 1500 \mathrm{~kg}$, and $2500 \mathrm{~kg}$ spacecraft respectively. Using Eqs. (23) - (27), the payload mass is plotted as a function of mission lifetime. 


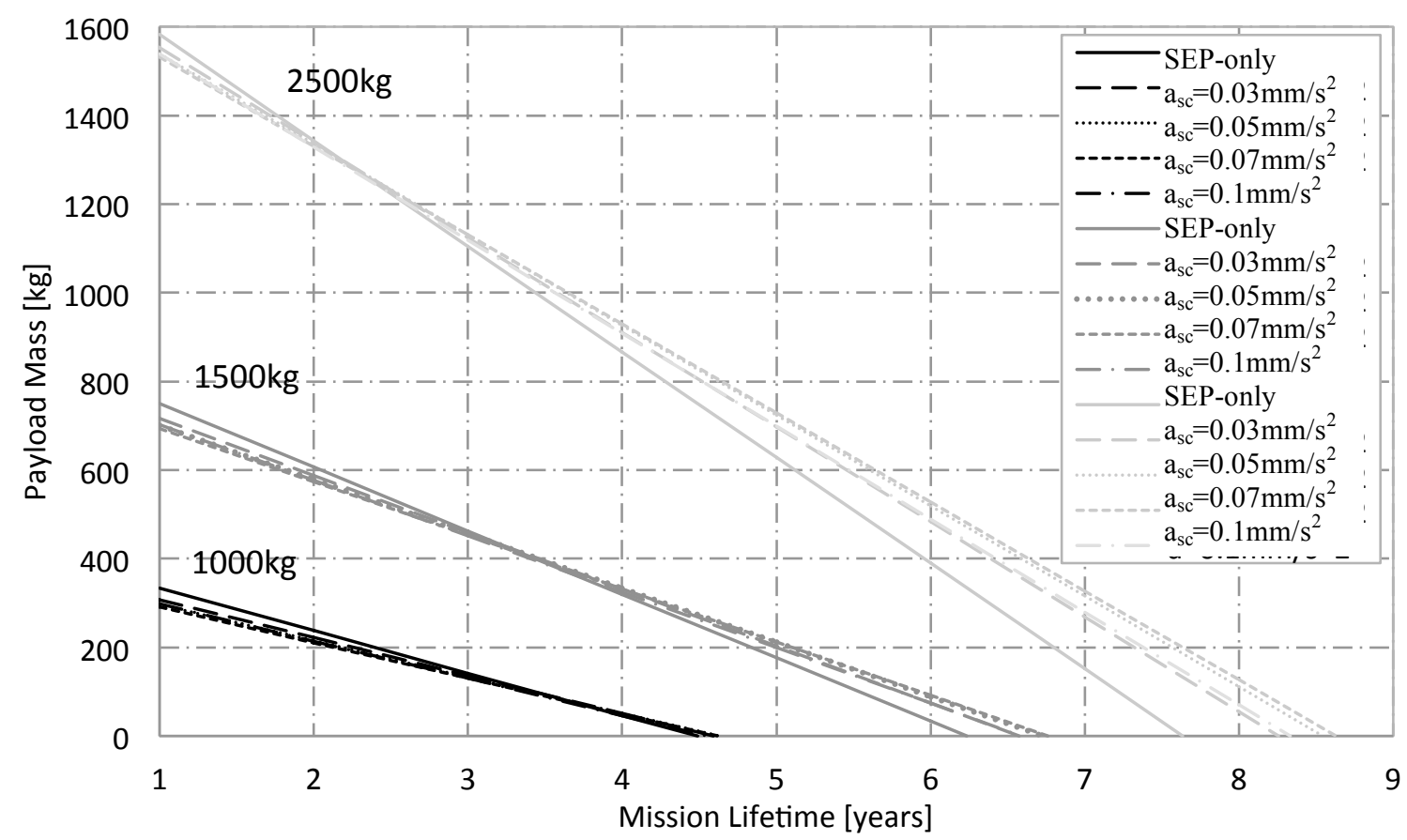

Fig. 9 Payload mass as a function of mission lifetime. Fixed launch mass.

Fig. 9 allows the maximum mission lifetime to be determined, that is, where there is no longer any capacity for useful payload. The maximum mission lifetimes for each initial spacecraft mass, for each of the solar sail characteristic accelerations considered are given in Table 7.

Table 7 Maximum mission lifetimes - fixed launch mass.

\begin{tabular}{cccc}
\hline \hline & & & \\
Characteristic Acceleration $\left[\mathbf{m m} / \mathbf{s}^{\mathbf{2}}\right]$ & $\mathbf{1 0 0 0 k g}$ & $\mathbf{1 5 0 0 k g}$ & $\mathbf{2 5 0 0 k g}$ \\
\hline SEP-only & 4.5 & 6.2 & 7.6 \\
$\mathbf{0 . 0 3}$ & 4.6 & 6.6 & 8.3 \\
$\mathbf{0 . 0 5}$ & 4.6 & 6.7 & 8.5 \\
$\mathbf{0 . 0 7}$ & 4.6 & 6.8 & 8.6 \\
$\mathbf{0 . 1 0}$ & 4.5 & 6.6 & 8.3 \\
\hline
\end{tabular}

Table 7 shows a modest increase in the Taranis mission lifetime with the addition of a solar sail to the system, with the maximum increase in lifetime of one year, occurring for the $2500 \mathrm{~kg}$ spacecraft with a solar sail characteristic acceleration of $0.07 \mathrm{~mm} / \mathrm{s}^{2}$. Table 7 shows that for the $1000 \mathrm{~kg}$ and $1500 \mathrm{~kg}$ spacecraft the increases in mission lifetime produced by the addition of the solar sail are negligible, with all increases less than half a year for all solar sails. Thus, it is shown from Table 7 and Eq. (9) - (10), that in order to make any significant increase in the lifetime of the Taranis mission, a large light solar sail is required, thus, it is expected that considerable development in solar sail technology is required. 


\section{Fixed Maximum Thrust}

As constraining the mass of the spacecraft allows very little increase in the lifetime of the Taranis missions without significant development of the solar sails, the scenario where the maximum thrust of the SEP system is the constraining parameter is now considered.

\section{Case 1}

As previously discussed, in this instance, the mass of each of the solar sails is assumed to equal the additional mass for the given thrust value (from Fig. 8). The payload mass as a function of mission lifetime is given in Fig. 10 for each of the thrust values considered. In this case the mission lifetime for each initial thrust is the same as the SEP- only lifetime regardless of the sail characteristic acceleration as all additional mass is used to add the sail and not to add any additional propellant.

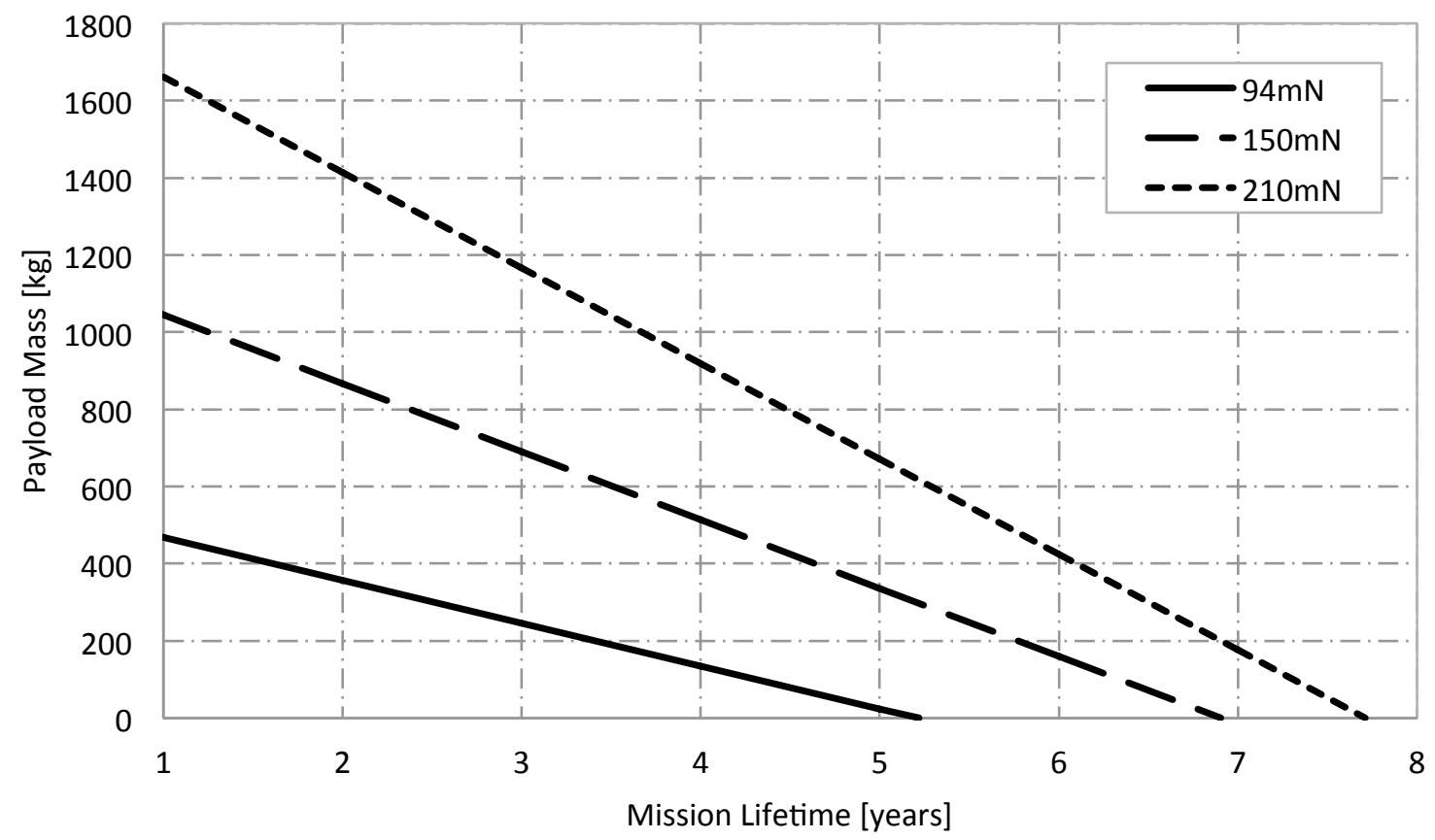

Fig. 10 Payload mass as a function of mission lifetime, case 1.

Fig. 10 shows maximum mission lifetimes of around 5.2 years, 6.9 years, and 7.7 years respectively for each of the maximum thrust values. Once again, Fig. 10 shows there is no increase in mission lifetime from the SEP-only case, when a solar sail is added, as the additional mass created by the lower acceleration is consumed fully by the solar sail and not for additional propellant. Thus, the solar sail technology required in this case is the base line, and to increase the mission lifetime an improvement in solar sail technology from these sails will be required. 


\section{Case 2}

Solar sail masses in this case are assumed to equal half of the additional mass and the remaining half of the additional mass is used to increase the capacity for useful payload. The resulting payload masses for each system are given in Fig. 11.

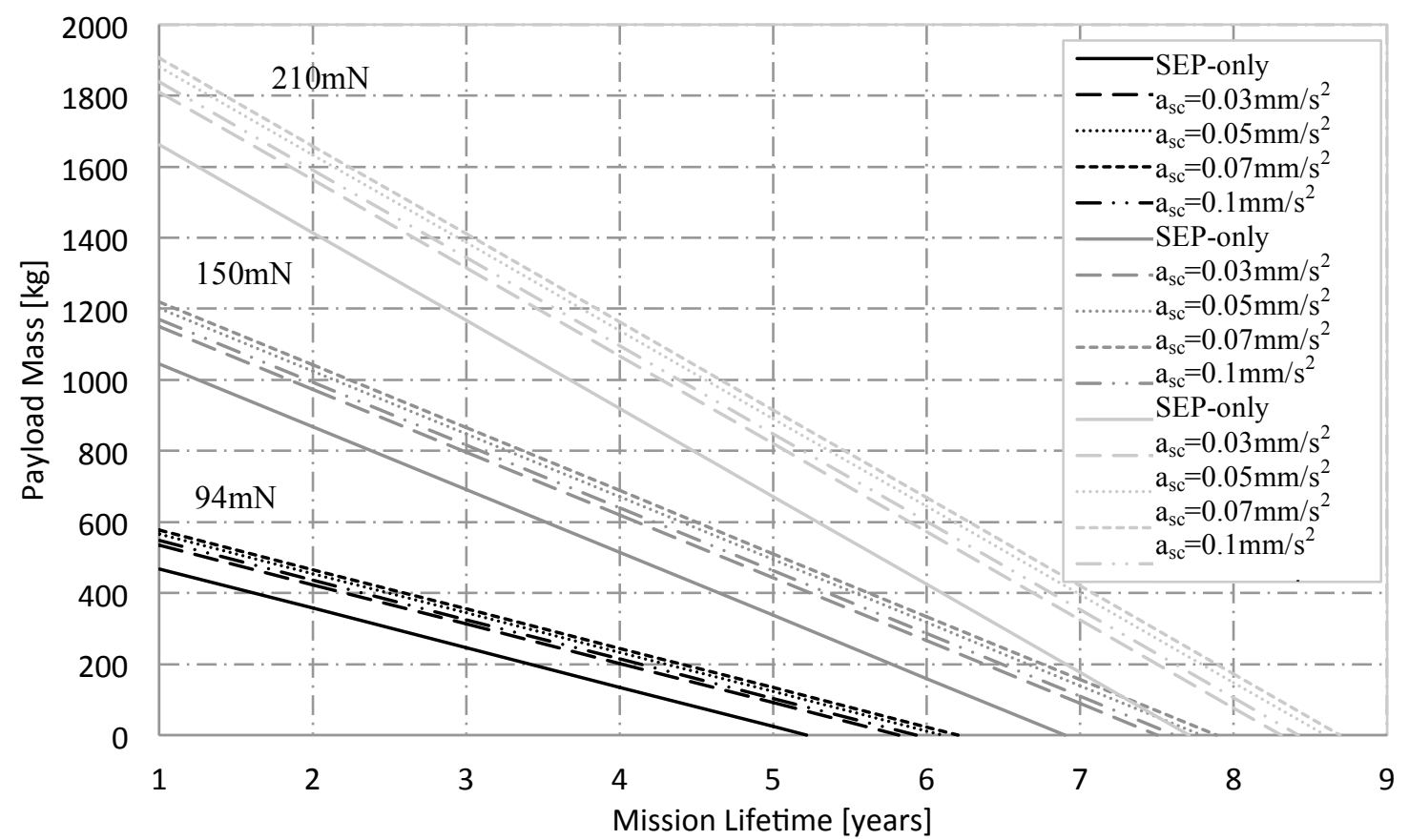

Fig. 11 Payload mass as a function of mission lifetime, for SEP-only and various solar sail characteristic accelerations.

Fig. 11 shows the increase in the mission lifetime for each of the hybrid solar sail / SEP systems considered for each of the initial thrust values. The maximum mission lifetimes are given in Table 8.

Table 8 Maximum mission lifetimes - fixed maximum thrust.

\begin{tabular}{cccc}
\hline \hline Characteristic Acceleration $\left[\mathbf{m m} / \mathbf{s}^{\mathbf{2}}\right]$ & $\mathbf{9 4} \mathbf{m N}$ & $\mathbf{1 5 0} \mathbf{m}$ & $\mathbf{2 1 0} \mathbf{N}$ \\
\hline $\mathbf{0}$ & 5.2 & 6.9 & 7.7 \\
$\mathbf{0 . 0 3}$ & 5.8 & 7.5 & 8.3 \\
$\mathbf{0 . 0 5}$ & 6.1 & 7.8 & 8.6 \\
$\mathbf{0 . 0 7}$ & 6.2 & 7.9 & 8.7 \\
$\mathbf{0 . 1 0}$ & 5.9 & 7.6 & 8.4 \\
\hline
\end{tabular}

Table 8 shows that although the maximum increase in mission lifetime is the same as the fixed launch mass case. However, more significant increases are shown for the smaller initial thrusts and for solar sails with smaller characteristic accelerations. In addition to the greater increase in mission lifetime, the solar sails required to achieve 
this increase are much heavier and are thus more feasible solutions. Where solar sails in the fixed launch mass case range between around $42 \mathrm{~kg}$ and $116 \mathrm{~kg}$, in the fixed thrust case 1 range from around $135 \mathrm{~kg}$ to $488 \mathrm{~kg}$ and in case 2 from $67 \mathrm{~kg}$ to $244 \mathrm{~kg}$.

\section{Thrust Range Analysis}

Although the Taranis orbit requires a constant acceleration, in reality as the propellant is consumed the mass of the spacecraft decreases, thus causing an increase in the acceleration from the SEP system. A variable thrust SEP system is therefore required. The thrust range necessary from the SEP system can be determined by finding the thrust at the beginning of the mission with all the propellant, and the thrust at the end of the mission with zero propellant. These thrust ranges are shown for a range of mission lifetimes for each of the hybrid systems and the SEP-only case for both the fixed launch mass case in Fig. 12, and the fixed maximum thrust case in Fig. 13.

Fixed Launch Mass

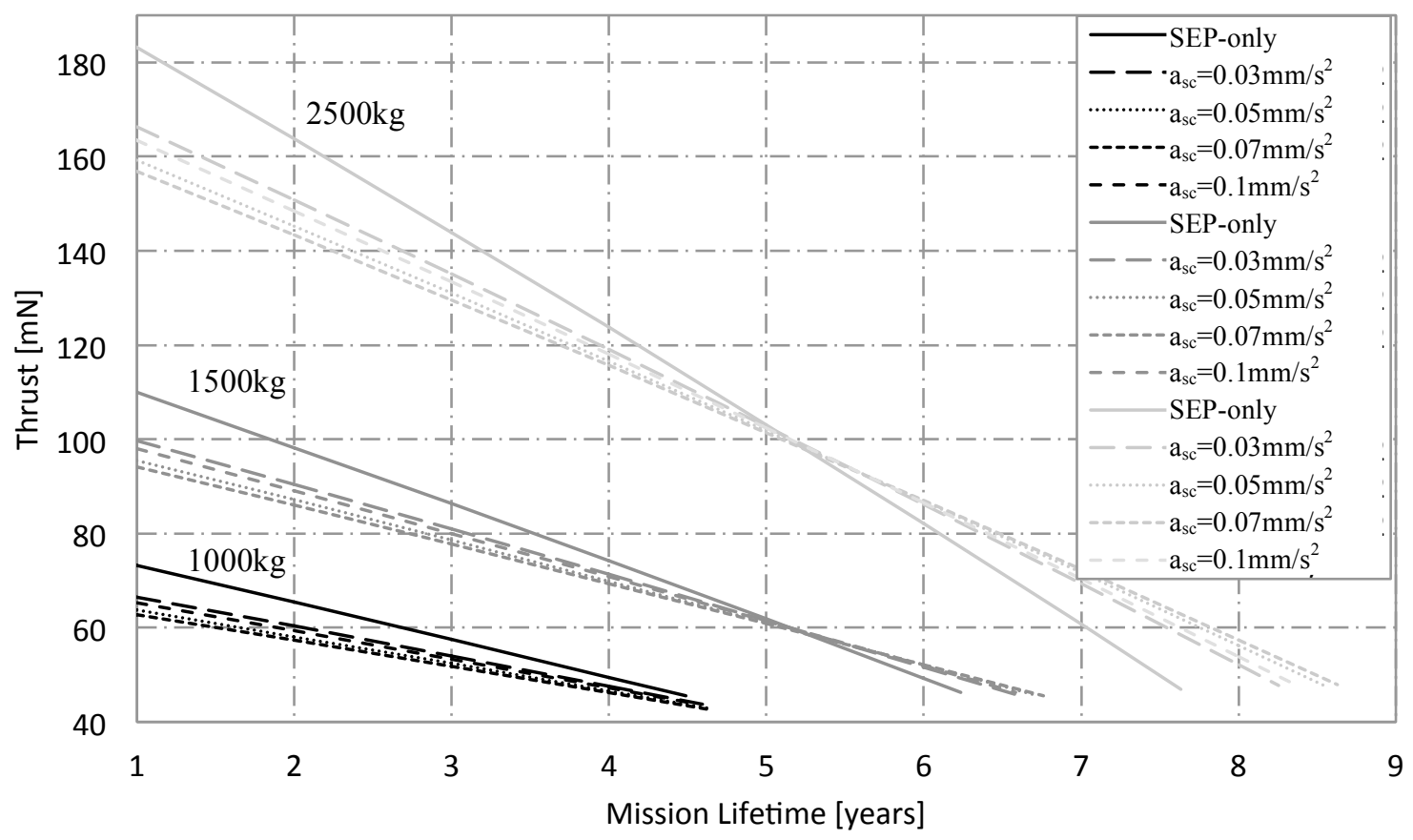

Fig. 12 Thrust ranges required by SEP system. Fixed launch mass.

In Fig. 12 it is shown that the addition of a solar sail to the SEP system decreases the thrust range required by the SEP system. For example, considering the $1500 \mathrm{~kg}$ initial thrust for a 4 year mission the SEP-only system requires $121.3 \mathrm{mN}$ at the beginning of the mission and $74.2 \mathrm{mN}$ at the end of the mission. This is compared with $102.2 \mathrm{mN}$ at 
the beginning of the mission and $69.4 \mathrm{mN}$ at the end of the four years for the solar sail of characteristic acceleration $0.07 \mathrm{~mm} / \mathrm{s}^{2}$

\section{Fixed Maximum Thrust}

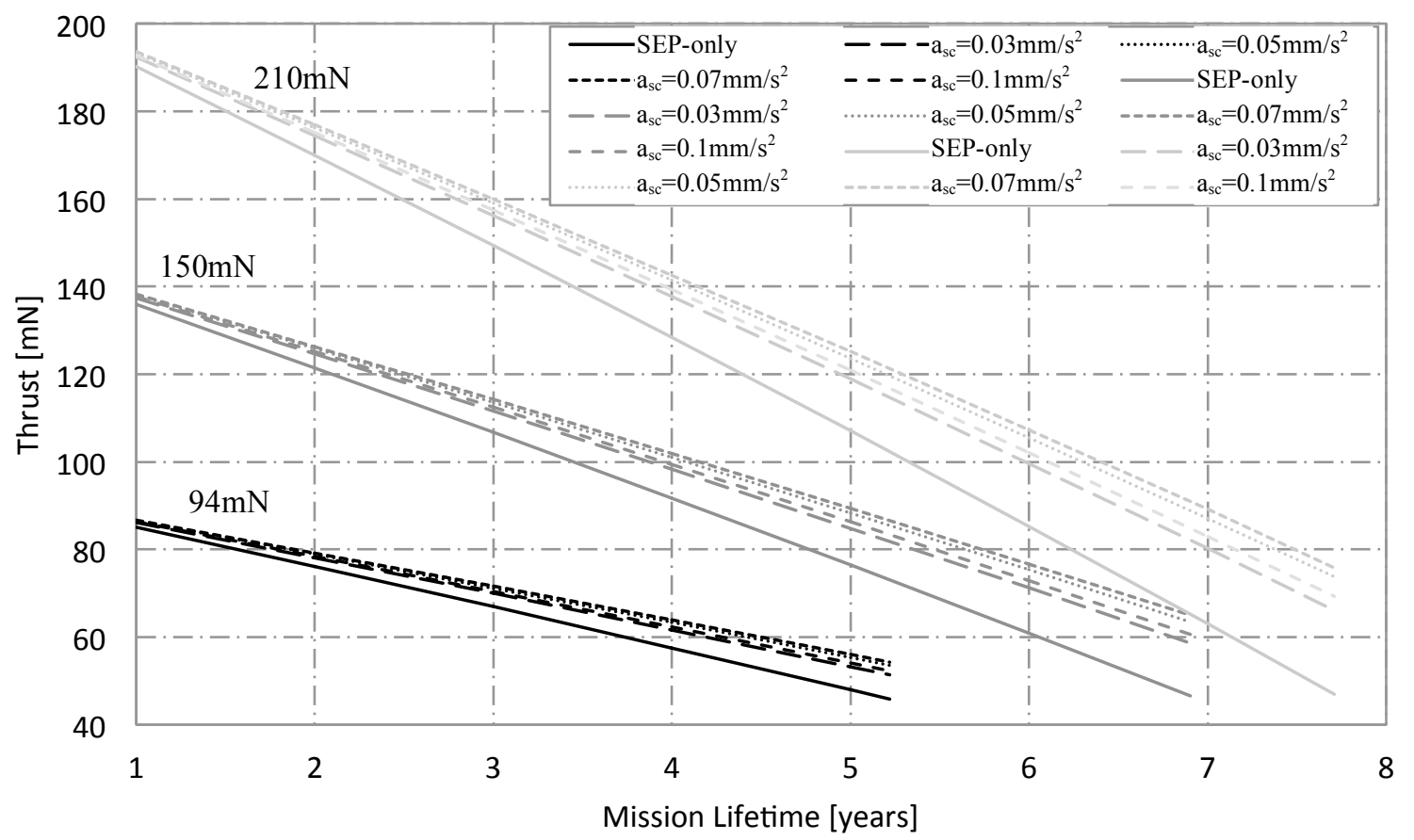

Fig. 13 Thrust ranges required by SEP system. Fixed maximum thrust.

Fig. 13 shows that, as in the fixed launch mass case, as larger solar sails are added to the system, the required SEP thrust range decreases. Considering the spacecraft with an initial thrust of $210 \mathrm{mN}$ and mission duration of 5 years, the SEP-only case has a final thrust of $107.1 \mathrm{mN}$. This is compared with final thrusts of $118.9 \mathrm{mN}, 123.6 \mathrm{mN}$, $125.1 \mathrm{mN}$, and $120.8 \mathrm{mN}$ respectively for each of the given solar sail characteristic accelerations.

\section{Technology Requirements}

Given that the parameters of the Taranis platform are defined for the mass and power required the systems and technology requirements of the platform can be investigated. Firstly, the requirements of the spacecraft including SEP thrusters, solar arrays and propellant tanks are discussed, followed by investigation of the possible solar sail design for each case discussed. 


\section{SEP Thrusters}

Assuming the total acceleration is constituted by two thrusters at any given time, one for each of the radial and transverse directions, the approximate required range per thruster for three mission durations, for the SEP-only case and the hybrid solar sail / SEP systems, are stated in Table 9.

\section{Fixed Launch Mass}

Table 9 Maximum and minimum thrust per thruster.

\begin{tabular}{|c|c|c|c|c|c|c|c|c|c|c|c|}
\hline \multirow[b]{2}{*}{$\begin{array}{c}\text { Initial } \\
\text { Mass } \\
{[\mathrm{kg}]}\end{array}$} & \multirow[b]{2}{*}{$\begin{array}{c}\text { Duration } \\
\text { [years] }\end{array}$} & \multicolumn{2}{|c|}{ SEP } & \multicolumn{2}{|c|}{$0.03 \mathrm{~mm} / \mathrm{s}^{2}$} & \multicolumn{2}{|c|}{$0.05 \mathrm{~mm} / \mathrm{s}^{2}$} & \multicolumn{2}{|c|}{$0.07 \mathrm{~mm} / \mathrm{s}^{2}$} & \multicolumn{2}{|c|}{$0.1 \mathrm{~mm} / \mathrm{s}^{2}$} \\
\hline & & $\begin{array}{c}\text { Max } \\
{[\mathrm{mN}]}\end{array}$ & $\begin{array}{c}\text { Min } \\
{[\mathrm{mN}]}\end{array}$ & $\begin{array}{c}\text { Max } \\
{[\mathbf{m N}]}\end{array}$ & $\begin{array}{c}\text { Min } \\
{[\mathrm{mN}]}\end{array}$ & $\begin{array}{c}\operatorname{Max} \\
{[\mathbf{m N}]}\end{array}$ & $\begin{array}{c}\text { Min } \\
{[\mathrm{mN}]}\end{array}$ & $\begin{array}{c}\operatorname{Max} \\
{[\mathrm{mN}]}\end{array}$ & $\begin{array}{c}\text { Min } \\
{[\mathbf{m N}]}\end{array}$ & $\begin{array}{c}\operatorname{Max} \\
{[\mathbf{m N}]}\end{array}$ & $\begin{array}{l}\text { Min } \\
{[\mathrm{mN}]}\end{array}$ \\
\hline 1000 & 3 & 40 & 29 & 36 & 27 & 35 & 26 & 34 & 26 & 36 & 27 \\
\hline 1000 & 4 & 40 & 25 & 36 & 24 & 35 & 23 & 34 & 23 & 36 & 24 \\
\hline 1500 & 4 & 61 & 37 & 54 & 36 & 52 & 35 & 51 & 35 & 53 & 35 \\
\hline 1500 & 6 & 61 & 25 & 54 & 26 & 52 & 26 & 51 & 26 & 53 & 26 \\
\hline 2500 & 4 & 101 & 62 & 91 & 60 & 87 & 58 & 85 & 58 & 89 & 59 \\
\hline 2500 & 6 & 101 & 41 & 91 & 43 & 87 & 43 & 85 & 44 & 89 & 43 \\
\hline 2500 & 7 & 101 & 30 & 91 & 35 & 87 & 36 & 85 & 36 & 89 & 35 \\
\hline
\end{tabular}

Table 9 demonstrates the reduction in thrust range required by the SEP thrusters for all of the hybrid systems proposed for each thruster. With the thrust range decreasing as the solar sail characteristic acceleration increases. All of the thrust ranges shown in Table 9 are achievable using current technology. The NSTAR thruster, which has undergone significant ground testing in addition to a flight test on the Deep Space 1 (DS1) spacecraft [16], is capable of providing between $20 \mathrm{mN}$ and $94 \mathrm{mN}$ of thrust. Thus, four NSTAR thrusters, one per required direction, are capable of providing the thrust range required for both the $1000 \mathrm{~kg}$ and $1500 \mathrm{~kg}$ initial masses, for all duration of mission considered, and for the $2500 \mathrm{~kg}$ spacecraft for all hybrid systems proposed. Furthermore, the QinetiQ T6 thruster, is throttleable between $30 \mathrm{mN}$ and $210 \mathrm{mN}$ [17] and is thus capable of providing the required thrust range for all of the mission durations for an initial mass of $2500 \mathrm{~kg}$ and for all systems for a 4 year mission for the $1500 \mathrm{~kg}$ spacecraft.

\section{Fixed Maximum Thrust}

Again, assuming the total acceleration is constituted by two thrusters at any given time, one for each of the radial and transverse directions, the approximate required range per thruster are stated in Table 10. 
Table 10 Maximum and minimum thrust per thruster.

\begin{tabular}{|c|c|c|c|c|c|c|c|}
\hline $\begin{array}{c}\text { Initial Thrust } \\
{[\mathrm{mN}]}\end{array}$ & $\begin{array}{l}\text { Duration } \\
\text { [years] }\end{array}$ & $\begin{array}{c}\text { Max Thrust } \\
{[\mathrm{mN}]}\end{array}$ & $\begin{array}{c}\text { SEP-only } \\
\text { Min Thrust } \\
{[\mathbf{m N}]}\end{array}$ & $\begin{array}{c}\mathrm{a}_{\mathrm{sc}}=0.03 \mathrm{~mm} / \mathrm{s}^{2} \\
\text { Min Thrust } \\
{[\mathrm{mN}]}\end{array}$ & $\begin{array}{c}\mathrm{a}_{\mathrm{sc}}=0.05 \mathrm{~mm} / \mathrm{s}^{2} \\
\text { Min Thrust } \\
{[\mathrm{mN}]}\end{array}$ & $\begin{array}{c}\mathrm{a}_{\mathrm{sc}}=0.07 \mathrm{~mm} / \mathrm{s}^{2} \\
\text { Min Thrust } \\
{[\mathrm{mN}]}\end{array}$ & $\begin{array}{c}\mathrm{a}_{\mathrm{sc}}=0.1 \mathrm{~mm} / \mathrm{s}^{2} \\
\text { Min Thrust } \\
{[\mathrm{mN}]}\end{array}$ \\
\hline 94 & 3 & 47 & 33 & 35 & 36 & 36 & 35 \\
\hline 94 & 4 & 47 & 29 & 31 & 32 & 32 & 31 \\
\hline 150 & 4 & 75 & 46 & 49 & 51 & 51 & 50 \\
\hline 150 & 6 & 75 & 30 & 36 & 38 & 38 & 36 \\
\hline 210 & 4 & 105 & 64 & 69 & 71 & 71 & 70 \\
\hline 210 & 6 & 105 & 43 & 50 & 53 & 54 & 51 \\
\hline 210 & 7 & 105 & 32 & 40 & 44 & 45 & 42 \\
\hline
\end{tabular}

Table 10 again demonstrates the reduction in thrust range required by the SEP thrusters for all of the hybrid systems proposed. It is shown that four NSTAR thrusters, one per required direction, are capable of providing the thrust range required for both the $94 \mathrm{mN}$ and $150 \mathrm{mN}$ initial thrust systems, for all duration of mission considered, for both SEP-only and hybrid systems. The QinetiQ T6 thruster is also capable of providing the required thrust range for all of the systems for all mission lifetimes with the exception of a 4 year mission using the SEP-only system with an initial thrust of $94 \mathrm{mN}$.

Solar Arrays

To enable the 90deg Taranis orbit, the power requirements of the spacecraft must be considered. The sizing of the required solar arrays is based on an end-of-life (EOL) solar array efficiency of 0.25 at 1 AU. The power required by each spacecraft of different initial thrust values, the mass of the solar arrays (from Eq.(26)) and the required solar array area, found using the Solar flux of $1370 \mathrm{~W} / \mathrm{m}^{2}$ at $1 \mathrm{AU}$, are given in Table 11 for the fixed launch mass and fixed maximum thrust.

\section{Fixed Launch Mass}

Table 11 Solar array sizing.

\begin{tabular}{ccccc}
\hline \hline $\begin{array}{c}\text { Characteristic } \\
\text { Acceleration }\left[\mathbf{m m} / \mathbf{s}^{\mathbf{2}}\right]\end{array}$ & $\begin{array}{c}\text { Initial Mass } \\
{[\mathbf{k g}]}\end{array}$ & $\begin{array}{c}\text { Maximum Power } \\
{[\mathbf{k W}]}\end{array}$ & $\begin{array}{c}\text { Solar Array Mass } \\
{[\mathbf{k g}]}\end{array}$ & $\begin{array}{c}\text { Solar Array Area } \\
{\left[\mathbf{m}^{\mathbf{2}}\right]}\end{array}$ \\
\hline SEP & 1000 & 1.7 & 38 & 5 \\
SEP & 1500 & 2.6 & 57 & 8 \\
SEP & 2500 & 4.3 & 94 & 13 \\
0.03 & 1000 & 1.5 & 34 & 4 \\
0.03 & 1500 & 2.3 & 51 & 7 \\
0.03 & 2500 & 3.8 & 85 & 11 \\
0.05 & 1000 & 1.5 & 32 & 4 \\
0.05 & 1500 & 2.2 & 48 & 6 \\
0.05 & 2500 & 3.6 & 81 & 11 \\
0.07 & 1000 & 1.4 & 32 & 4
\end{tabular}




\begin{tabular}{ccccc}
0.07 & 1500 & 2.1 & 48 & 6 \\
0.07 & 2500 & 3.6 & 80 & 11 \\
0.1 & 1000 & 1.5 & 33 & 4 \\
0.1 & 1500 & 2.2 & 50 & 6 \\
0.1 & 2500 & 3.7 & 83 & 11 \\
\hline
\end{tabular}

Table 11 shows the modest reduction in both mass and required area of the solar arrays by the use of hybrid solar sail / SEP system, with the required area decreasing as the solar sail characteristic acceleration is increased. Results show that the required sizes of the solar arrays, for all cases, are modest and feasible using current solar array technology. With the solar arrays of Rosetta totaling $61.5 \mathrm{~m}^{2}$ [20] and SMART-1's arrays having an area of $10 \mathrm{~m}^{2}$ [21] for spacecraft of $3000 \mathrm{~kg}$ and $370 \mathrm{~kg}$ respectively.

\section{Fixed Maximum Thrust}

Table 12 Solar array sizing - fixed maximum thrust.

\begin{tabular}{cccc}
\hline \hline & & & \\
Initial Thrust $[\mathbf{m N}]$ & Maximum Power $[\mathbf{k W}]$ & Solar Array Mass $[\mathbf{k g}]$ & Solar Array Area $\left[\mathbf{m}^{2}\right]$ \\
\hline 94 & 2.0 & 44 & 6 \\
150 & 3.2 & 70 & 9 \\
210 & 4.4 & 98 & 13 \\
\hline \hline
\end{tabular}

Table 12 shows that, as with the fixed launch mass case, the required sizes of the solar arrays, for all cases, are feasible using current solar array technology.

\section{Propellant Tanks}

Finally, the storage requirements for the requisite propellant mass for given mission durations are examined. The propellant mass for each initial thrust value considered are determined using Eq. (27) and are shown in Table 13 Table 14.

Table 13 Propellant mass - fixed launch mass.

\begin{tabular}{ccccccc}
\hline \hline $\begin{array}{c}\text { Initial } \\
\text { Mass [kg] }\end{array}$ & $\begin{array}{c}\text { Mission Duration } \\
\text { [years] }\end{array}$ & $\begin{array}{c}\text { SEP-only } \\
\text { Propellant } \\
\text { Mass [kg] }\end{array}$ & $\begin{array}{c}\mathrm{a}_{\mathrm{sc}}=0.03 \mathrm{~mm} / \mathrm{s}^{2} \\
\text { Propellant } \\
\text { Mass [kg] }\end{array}$ & $\begin{array}{c}\mathrm{a}_{\mathrm{sc}}=0.05 \mathrm{~mm} / \mathrm{s}^{2} \\
\text { Propellant } \\
\text { Mass [kg] }\end{array}$ & $\begin{array}{c}\mathrm{a}_{\mathrm{sc}}=0.07 \mathrm{~mm} / \mathrm{s}^{2} \\
\text { Propellant } \\
\text { Mass [kg] }\end{array}$ & $\begin{array}{c}\mathrm{a}_{\mathrm{sc}}=0.1 \mathrm{~mm} / \mathrm{s}^{2} \\
\text { Propellant } \\
\text { Mass [kg] }\end{array}$ \\
\hline 1000 & 4 & 347 & 311 & 297 & 292 & 305 \\
1500 & 6 & 780 & 700 & 668 & 657 & 687 \\
2500 & 7 & 1578 & 1362 & 1298 & 1277 & 1335 \\
\hline \hline
\end{tabular}

Table 13 shows the reduced propellant mass for the hybrid solar sail / SEP systems. The NASA Dawn mission Xenon tanks have a capacity of $425 \mathrm{~kg}$ of propellant, thus the Taranis spacecraft (both SEP-only and hybrid systems), propellant mass requirements can be accommodated using a single propellant tank for a four year mission. 
For a six year mission this is increased to two tanks for all proposed systems. Finally, four tanks are required for all seven year missions. The single tank volume is $0.27 \mathrm{~m}^{3}$, so that the equivalent tank radius for a spherical tank is $0.4 \mathrm{~m}$, thus the total propellant mass requirements for the possible Taranis platforms can be accommodated in a modest volume. Note that results are included for the $0.1 \mathrm{~mm} / \mathrm{s}^{2}$ solar sail are included to show the increase in propellant mass from the $0.07 \mathrm{~mm} / \mathrm{s}^{2}$ sail.

Table 14 Propellant mass - fixed maximum thrust.

\begin{tabular}{ccc}
\hline \hline & & \\
Initial Thrust [mN] & Mission Duration [years] & Propellant Mass [kg] \\
\hline 94 & 4 & 403 \\
150 & 6 & 965 \\
210 & 7 & 1576 \\
\hline \hline
\end{tabular}

Table 14 shows that the propellant mass requirements can be accommodated using a single propellant tank for a four year mission and, three propellant tanks for the six year mission and finally four tanks for the seven year missions.

Solar Sail

Following design of the spacecraft components, possible design of the solar sails is considered. Sizing of the solar sail is conducted to determine the technology development, if any, needed to allow the proposed hybrid lowthrust propulsion missions to become feasible.

\section{Fixed Launch Mass}

The design space for each of the solar sails for an initial mass of 1000kg is shown in Fig. 14. This gives the required area, found using Eq.(11), for each sail of a given characteristic acceleration, varying the sail loading and assuming a sail efficiency of 0.85 [12]. 


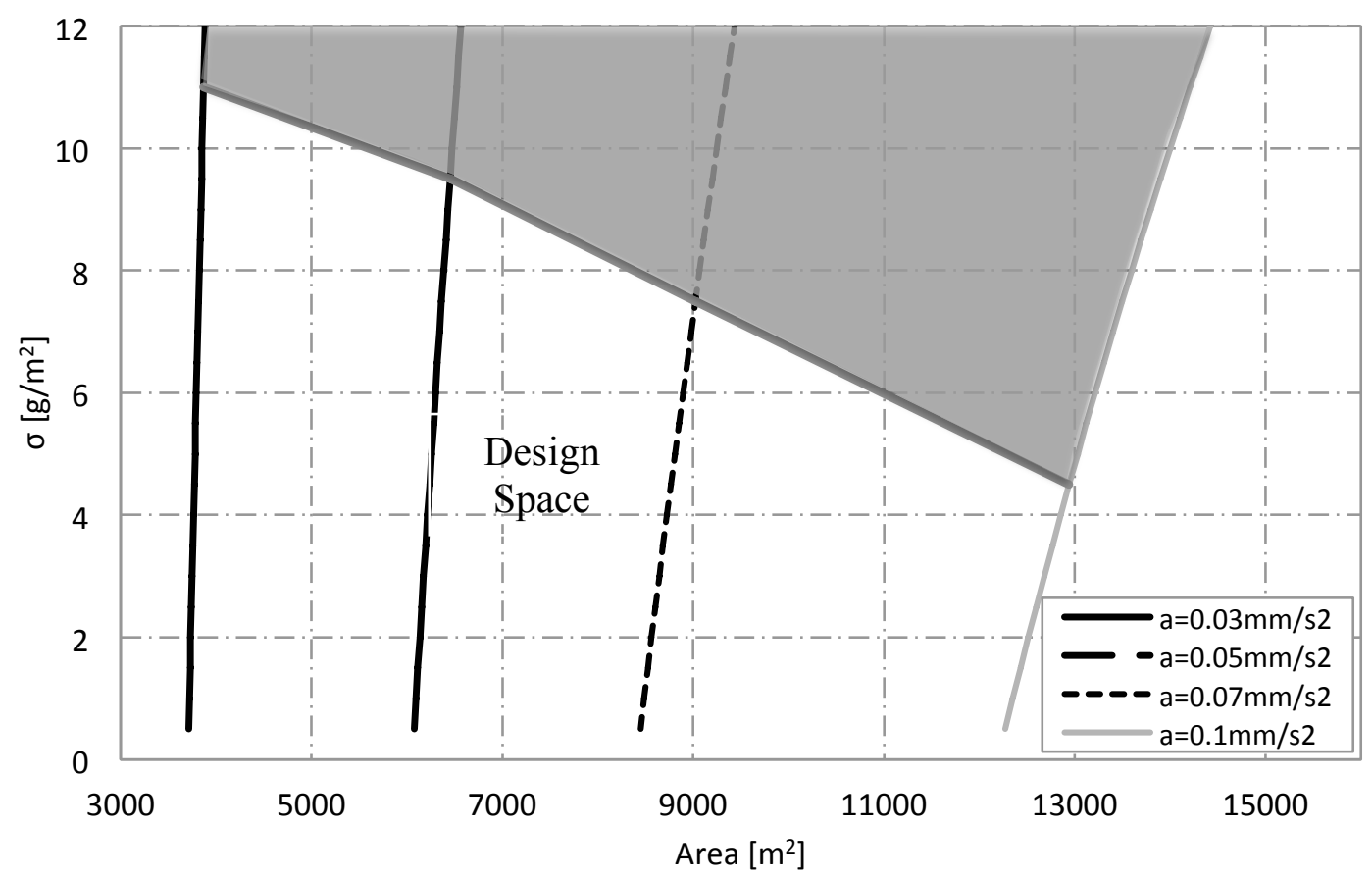

Fig. 14 Design space for various characteristic accelerations. Fixed launch mass.

Fig. 14 shows the design space for each of the solar sails considered, where below the shaded area the total spacecraft mass is below the initial mass value, thus the shaded area bounds the design limit of the sail. Similar figures can also be plotted for the initial mass values of $1500 \mathrm{~kg}$ and $2500 \mathrm{~kg}$, although these are not included within the paper. However, these figures show an increase in solar sail area and a significant decrease in sail loading as the initial spacecraft mass increases. Thus, significant development in solar sail technology is required to produce larger, lighter sails to make these hybrid solar sail / SEP systems feasible.

\section{Fixed Maximum Thrust}

Case 1

The design space for each of the solar sails for an initial thrust of $94 \mathrm{mN}$, are given in Fig. 15, giving the required solar sail area, again assuming a sail efficiency of 0.85 [12]. 


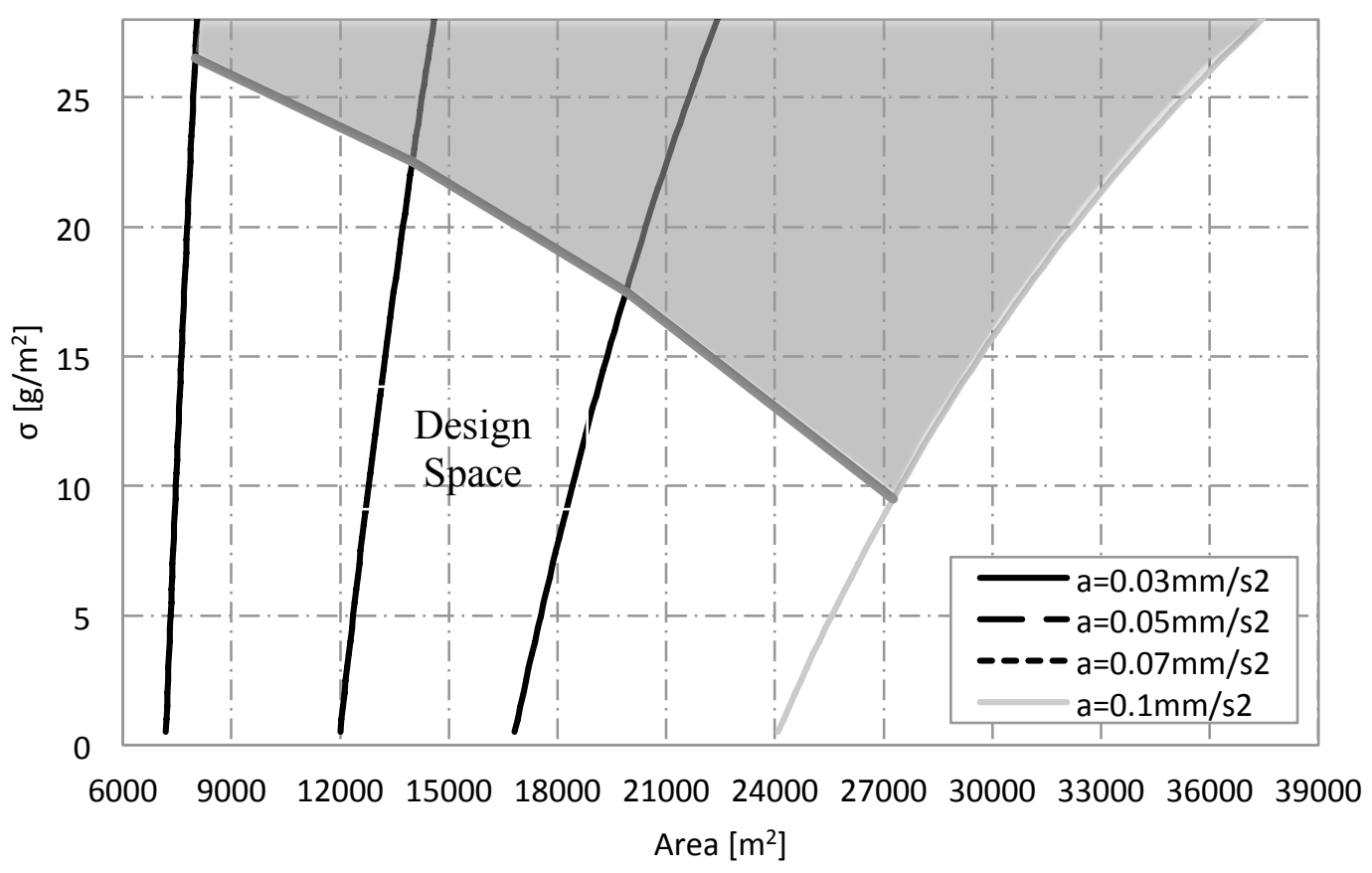

Fig. 15 Design space for various characteristic accelerations. Fixed maximum thrust, case 1.

Fig. 15 shows the design space for each of the solar sails considered for a $94 \mathrm{mN}$ initial thrust, where once again the shaded area bounds the design limit. It is shown that although the sail area is of the same order of magnitude as those required for the fixed mass case, the sail loading is significantly higher. Thus, the solar sails in this case are within near or mid-term technology. Similar figures can also be plotted for the initial thrust values of $150 \mathrm{mN}$ and $210 \mathrm{mN}$, although these are not included within the paper. However, these figures show that the required area of the solar sail increases as the initial thrust is increased.

Case 2

In this instance lighter solar sails half of the additional mass are considered for each of the given characteristic accelerations, again assuming an efficiency of 0.85 [12] and varying the sail loading to determine the requisite area, the results for an initial thrust of $94 \mathrm{mN}$ are given in Fig. 16 . 


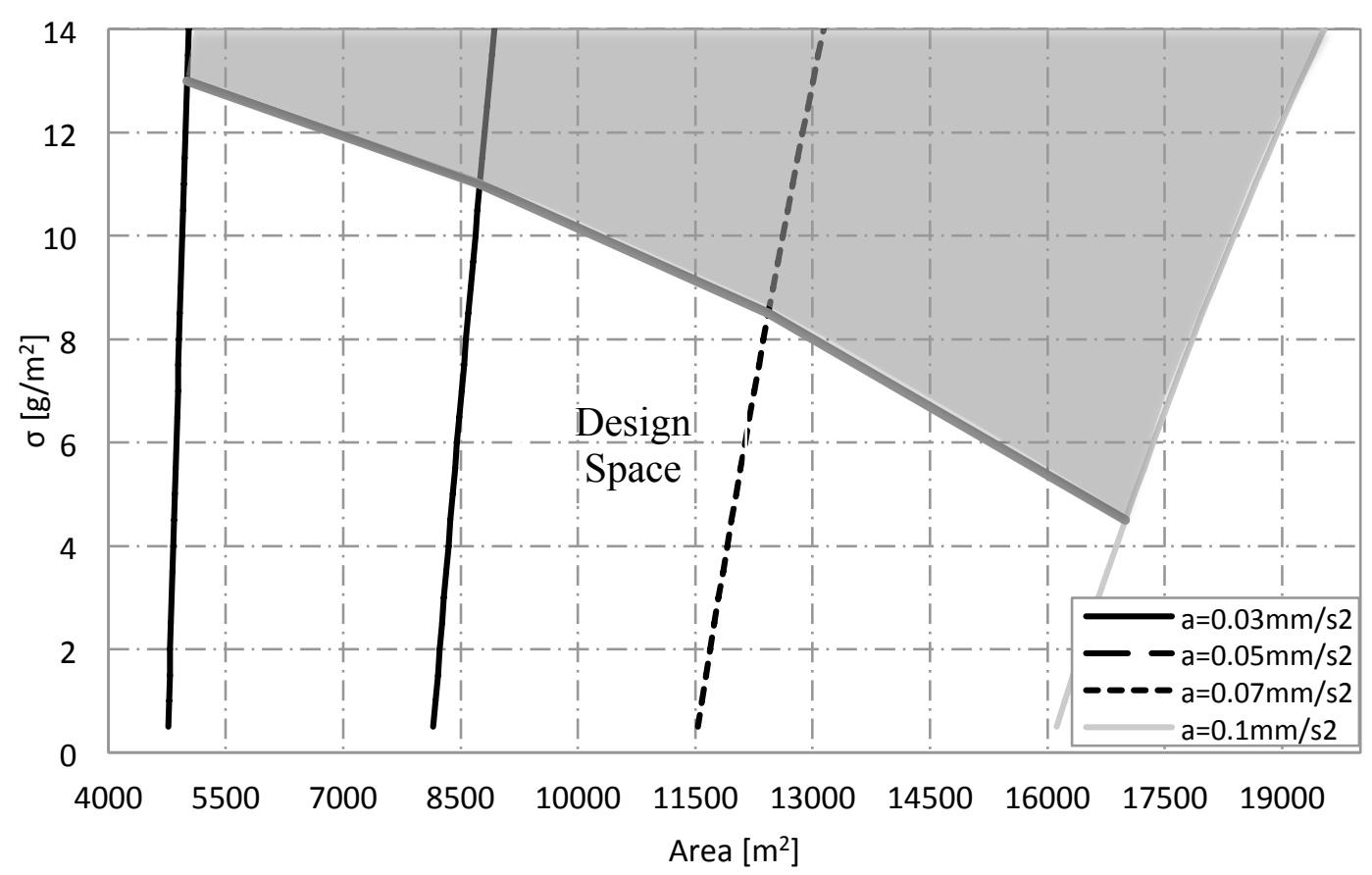

Fig. 16 Design space for various characteristic accelerations. Fixed maximum thrust, case 2.

Comparison of design space charts from case 1 and case 2 shows that although the required areas of the sails are of the same order of magnitude, the solar sails in case 2 are significantly lighter. Thus, to enhance the lifetime of the Taranis mission developments in solar sail technology are required to produce these relatively large, light sails. Similarly, plots of the solar sail design space for the initial thrust values of $150 \mathrm{mN}$ and $210 \mathrm{mN}$ can be plotted and again show a significant increase in the required area from the $94 \mathrm{mN}$ solution.

\section{Conclusion}

Hybrid solar sail and solar electric propulsion systems have been considered to maintain the novel, 12-hr, highly elliptical orbit, inclined at 90deg to the equator, termed a Taranis orbit. This hybrid propulsion lowers the demand on the solar electric propulsion system by reducing the amount of propellant consumed, thus increasing the possible lifetime of the mission and the capacity for useful payload. The addition of a solar sail to the solar electric propulsion system could lower the advanced degree of difficulty of the solar sail, while the electric thruster compensates for the solar sail's inability to thrust in the direction of the Sun. However, in some scenarios it may be counter productive to include a sail and therefore may offer little or no benefit, and the addition of a solar sail to the system could lead to system level design complexities, which are not considered in this paper. Two constraining 
parameters were considered, firstly the case where the launch mass of the spacecraft is fixed, and secondly where the maximum thrust of the solar electric propulsion thruster constrains the system. When the launch mass of the spacecraft is fixed, the increase in the mission lifetime from the pure electric propulsion system is negligible. The solar sails required to achieve these increases in lifetime are extremely large, light sails, and thus, considerable developments in solar sail technology are necessary to make these missions feasible. In the case of the fixed maximum thrust, the increase in mission lifetime is greater than that achieved using a fixed launch mass. In addition to this, the physical size of the solar sails required are of the same order of magnitude as the fixed launch mass sails, however, these are much heavier sails making them more feasible solutions. It has therefore been shown that the gain from the addition of a solar sail to the system is very little, unless the mass of the spacecraft is increased.

\section{References}

1. Wertz, J. R., "Mission Geometry: Orbit and Constellation Design and Management”, The Space Technology Library, ed., Vol., 2001. p. 619.

2. Anderson, P., and Macdonald, M. "Extension of Earth Orbits Using Low-Thrust Propulsion," 61st International Astronautical Congress. Prague, Czech Republic, 2010.

3. Anderson, P., and Macdonald, M. "Extension of the Molniya Orbit Using Low-Thrust Propulsion,"21st AAS/AIAA Spaceflight Mechanics Meeting,New Orleans, LA,2011

4. Anderson, P., and Macdonald, M. "Extension of Highly Elliptical Earth Orbits using Continuous Low-Thrust Propulsion," Journal Guidance Control and Dynamics, 2011, Accepted November 2011

5. Macdonald, M., and McInnes, C. R. "Solar Sail Science Mission Applications and Advancement," Advances in Space Research Vol. In Press, 2011,

6. Macdonald, M., "Solar Sailing: Applications and Technology Advancement”, ed. Hall, J., Vol., InTech, 2011. p.

7. Heiligers, J. "Displaced Geostationary Orbits using Hybrid Low-Thrust Propulsion,"61st International Astronautical Congress,Prague, CZ,2010,27th September - 1st October

8. McKay, R., Macdonald, M., Biggs, J., and McInnes, C. R. "Survey of Highly Non-Keplerian Orbits With Low-Thrust Propulsion," Journal of Guidance, Control and Dynamics Vol. 34, No. 3, 2011, pp. 645

9. Ceriotti, M., and McInnes, C. R. "A Near Term Pole-Sitter using Hybrid Solar Sail Propulsion,"2nd International Symposium on Solar Sailing,New York, USA,2010,July 20-22 2010

10. Ceriotti, M., and McInnes, C. R. "An Earth Pole-Sitter using Hybrid Propulsion,"AIAA/AAS Astrodynamics Specialist Conference,Toronto, Canada,2010,2nd August - 5th August 2010 
11. Ceriotti, M., and McInnes, C. R. "Hybrid Solar Sail and SEP Propulsion for Novel Earth Observation Missions.,"61st International Astronautical Congress,Prague, CZ,2010,27 September - 1 October 2010

12. McInnes, C. R., "Solar Sailing Technology, Dynamics and Mission Applications", ed. Publishing, S. P., Vol., Chichester UK, 1999. p. 14,40,115,58.

13. Macdonald, M., and McInnes, C. R. "Analytical Control Laws for Planet-Centered Solar Sailing," Journal of Guidance, Control and Dynamics Vol. 28, No. 5, 2005, pp. 1038September - October 2005

14. Macdonald, M. "Analytical Methodologies for Solar Sail Trajectory Design," Department of Aerospace Engineering. Vol. Doctor of Philosophy, Univeristy of Glasgow, Glasgow, 2005.

15. Green, A. J. "Optimal Escape Trajectories from a High Earth Orbit by use of Solar Radiation Pressure," Department of Aeronautics and Astronautics. Vol. Master of Science, Massachusetts Institude of Technology, 1977.

16. Brophy, J. R., Kakuda, R. Y., and Polk, J. E. "Ion Propulsion System (NSTAR) DS1 Technology Validation Report."

17. Wallace, N. C. "Testing of The QinetiQ T6 Thruster in Support of the ESA BepiColombo Mercury Mission," 4th International Spacecraft Propulsion Conference (ESA SP-55). Cagliari, Sardinia, Italy, 2004.

18. Brophy, J. R. "Advanced Ion Propulsion Systems for Affordable Deep-Space Missions," Acta Astronautica Vol. 52, 2003, pp. 309

19. Wertz, J. R., and Larson, W. J., "Space Mission Analysis and Design", ed., Vol. Third Edition, Microcosm Press and Kluwer Academic Publishers, 1999. p. 333.

20. D'Accolti, G., Beltrame, G., Ferrendo, E., Brambilla, L., Contini, R., Vallini, L., Mugnuolo, R., Signorini, C., Caon, A., and Fiebrich, H. "The Solar Array Photovoltaic Assembly for the Rosetta Orbiter and Lander Spacecrafts," Sixth European Conference. Porto, Portugal, 2002.

21. Racca, G. D., Marini, A., Stagnaro, L., Van Dooren, J., Di Napoli, L., Foing, B. H., Lumb, R., Volp, J., Brinkmann, J., Grunagel, R., Estublier, D., Anflo, K., Berge, S., Bodin, P., Edfors, A., Hussain, A., Kugelberg, J., Larsson, N., Ljung, B., Meijer, L., Mortsell, A., Nordeback, T., Persson, S., and Sjoberg, F. "SMART-1 Mission Description and Development Status," Planetary and Space Science Vol. 50, No. 14-15, 2002, p. 1323 